Illinois State University

ISU ReD: Research and eData

Theses and Dissertations

3-10-2020

\title{
The Water Is Always Running: Vaporwave, Fluxus, And The Role Of Defamiliarization In Music-Led Virtual Realities
}

Zachary William Buckley

Illinois State University, zwbuckley@icloud.com

Follow this and additional works at: https://ir.library.illinoisstate.edu/etd

Part of the Computer Sciences Commons, and the Fine Arts Commons

\section{Recommended Citation}

Buckley, Zachary William, "The Water Is Always Running: Vaporwave, Fluxus, And The Role Of Defamiliarization In Music-Led Virtual Realities" (2020). Theses and Dissertations. 1215.

https://ir.library.illinoisstate.edu/etd/1215

This Thesis is brought to you for free and open access by ISU ReD: Research and eData. It has been accepted for inclusion in Theses and Dissertations by an authorized administrator of ISU ReD: Research and eData. For more information, please contact ISUReD@ilstu.edu. 
THE WATER IS ALWAYS RUNNING: VAPORWAVE, FLUXUS, AND THE ROLE OF

DEFAMILIARIZATION IN MUSIC-LED VIRTUAL REALITIES

\section{ZACHARY WILLIAM BUCKLEY}

77 Pages

This thesis examines how a Fluxus approach to participatory music can leverage environmental metaphors and affordances, heighten user awareness of their interactions through a defamiliarized approach to musical interaction, and balance functional and creative user experiences in a meaningful and participatory musical interaction. This research has been conducted using the virtual reality experience The Water Is Always Running. This work presents an unusual music-making environment, a 3D kitchen with dishwashing simulation, to explore how a defamiliarized approach to musical interaction and participation can heighten the awareness of process for the user. To create this work, ideas have been leveraged from acoustic ecology, vaporwave, virtual reality musical instrument design, and human-centered design.

KEYWORDS: Music Composition; Virtual Reality; Fluxus; Acoustic Ecology 
THE WATER IS ALWAYS RUNNING: VAPORWAVE, FLUXUS, AND THE ROLE OF DEFAMILIARIZATION IN MUSIC-LED VIRTUAL REALITIES

\section{ZACHARY WILLIAM BUCKLEY}

A Thesis Submitted in Partial Fulfillment of the Requirements for the Degree of

MASTER OF SCIENCE

Wonsook Kim School of Art

ILLINOIS STATE UNIVERSITY 
Copyright 2020 Zachary William Buckley 
THE WATER IS ALWAYS RUNNING: VAPORWAVE, FLUXUS, AND THE ROLE OF DEFAMILIARIZATION IN MUSIC-LED VIRTUAL REALITIES

ZACHARY WILLIAM BUCKLEY

COMMITTEE MEMBERS:

Kristin Carlson, Chair

Roy Magnuson

Matthew Smith 


\section{ACKNOWLEDGMENTS}

To my committee members, Roy Magnuson and Matthew Smith, I am incredibly thankful for the wealth of feedback and encouragement you all have provided me throughout this process. Each of you trusted me completely when I told you that a dishwashing simulator was my grand idea and for that I will always be grateful.

To Annie Sunjakun and M. Anthony Reimer, thank you both for taking such an eager interest in my research. Your suggestions and wealth of knowledge have pushed my work in directions that would not have been possible before.

To Aaron Paolucci and my family in the Program in Creative Technologies, thank you for letting me undertake this journey. It is one of my deepest honors to have been able to be the first M.S. Creative Technologies candidate to write a thesis. Beyond that, it was a great comfort knowing I had the full support of the program behind me during this process.

To my chair, Kristin Carlson, you have been the greatest champion I could have ever asked for. Your support, guidance, and endless enthusiasm for my ideas and research have been the cornerstone of this experience for me. You've always met me exactly where I'm at and you've always been ready to help me find a way forward any of the many times that I've found myself stumped throughout this process. Thank you so much.

And, finally, to my wife, Emma, you are the unsung hero of this whole journey. Everybody should know how patiently you've listened to me discuss washing dishes in virtual reality all while I simultaneously complain any time that I have to wash dishes in this reality. That is an irony that transcends the concept of reality entirely and you, my love, have handled it with such effortless grace. And for that and your endless support, I thank you.

\section{Z.W.B.}




\section{CONTENTS}

Page

ACKNOWLEDGMENTS

TABLES $\quad$ iv

FIGURES

CHAPTER I: INTRODUCTION 1

CHAPTER II: LITERATURE REVIEW

$\begin{array}{ll}\text { Acoustic Ecology } & 7\end{array}$

$\begin{array}{ll}\text { Vaporwave } & 10\end{array}$

Fluxus, Defamiliarization, and Human-Centered Design 12

Virtual Reality Musical Instruments and Visual Design for Computer Music 15

Metaphors, the Virtual Environment, and Embodiment 16

CHAPTER III: THE WATER IS ALWAYS RUNNING 19

Project Description $\quad 19$

$\begin{array}{ll}\text { Design Process } & 22\end{array}$

$\begin{array}{ll}\text { Interaction Considerations } & 22\end{array}$

$\begin{array}{ll}\text { Musical Considerations } & 26\end{array}$

$\begin{array}{ll}\text { Environmental Considerations } & 31\end{array}$

Supporting Design Through Conceptual Vision 33

CHAPTER IV: STUDY AND DATA ANALYSIS 36 
Study Design

$\begin{array}{ll}\text { Major Themes } & 39\end{array}$

$\begin{array}{ll}\text { Environment } & 40\end{array}$

$\begin{array}{ll}\text { Interaction } & 42\end{array}$

Constraints and limitations. $\quad 43$

$\begin{array}{ll}\text { Music } & 45\end{array}$

$\begin{array}{ll}\text { Emotional Responses } & 50\end{array}$

$\begin{array}{ll}\text { Overall Experience } & 52\end{array}$

CHAPTER V: DISCUSSION

Key Findings $\quad 55$

Converge the Functional and the Creative 56

$\begin{array}{ll}\text { Subvert Expectations } & 58\end{array}$

$\begin{array}{ll}\text { Design to Flip the Switch } & 60\end{array}$

$\begin{array}{ll}\text { Create an Acoustic Ecology } & 62\end{array}$

User Agency is the Score / Environment is the Instrumentation 65

$\begin{array}{ll}\text { Limitations and Future Works } & 67\end{array}$

CHAPTER VI: CONCLUSION

$\begin{array}{lc}\text { REFERENCES } & 71\end{array}$

APPENDIX A: INTERVIEW QUESTIONS ASKED TO STUDY PARTICIPANTS 77

iii 


\section{TABLES}

Table

Page

1. Acoustic Ecology Terms, The Handbook for Acoustic Ecology

2. Participant Responses to Establishing Questions 


\section{FIGURES}

Figure $\quad$ Page

1. User view of The Water Is Always Running 19

2. Early development image showing use of Leap Motion Controller 20

3. Layout of The Water Is Always Running 21

4. Early mapping schemata for musical interactions in The Water Is Always Running 28

5. Visualization of music playback speed mapped to dish placement along Y-axis 29

6. Final mapping schemata for musical interactions in The Water Is Always Running 30

7. Participant 2 throwing dishes into the air 44

8. Participant 3 working through the dishes 51 


\section{CHAPTER I: INTRODUCTION}

Composer R. Murray Schafer suggests that people listen to their environment as if it were a piece of music, and advocates that people take responsibility for its composition (The Tuning of the World 1977). Historically, Shafer's concern has been primarily for the relationship between people and their physical environment. Through his work and advocacy, he is the father of acoustic ecology, a rich discipline that is concerned with sound as a mediating force between humans and their environment. While Shafer's provocative charge was issued for the physical world, I am interested in applying it to virtual worlds of my own composition. I am not alone in this interest. As excitement has grown for virtual reality, thanks to recent developments in increasingly affordable and user-friendly hardware, so too has the call for audio-first virtual reality experiences ("FAVR 2019: Future of Audio in Virtual Reality," 2019) (“Sonic Interactions in Virtual Environments," 2020).

A great challenge when building virtual sonic worlds, beyond simply creating a piece of music, the composer is also creating a world. Regardless of whether the VR experience a composer is creating is entirely aural or aural/visual, the end result is the same: to transport the audience into a new space that occupies a world separate from the physical world in which they reside. The composer interested in composing musical worlds as audio-first or music-led virtual realities now finds a host of other concerns. Is this world interactive or linear? As audiences interact with this musical world, is it challenging or engaging enough to hold their attention? Are the present interactions understandable in a way that allows the audience to reach the conclusion of the musical idea? How does the composer balance authorial intent with any audience desire to interact and play within their virtual world? These are questions and challenges many composers do not necessarily face when sitting down to write a piece of music. 
These questions allude to the fact that the composer of music-led virtual realities must now be a designer as well. In a music-led virtual reality, the musical content is meaningless if it cannot be accessed. If a composer designs interactions that are too challenging, unrewarding, or difficult to understand then at best the audience loses immersion and at worst they abandon the experience altogether because of a lack of interest or ability to proceed. If each of these inaccessible interactions contains a phrase or passage of music, then the audience is left with an incomplete experience that is less than the composer intended. This imparts an important point: before a music-led virtual reality can make musical sense, it must first make human sense.

Just as virtual reality is a medium that draws on a multitude of unique disciplines, so can the composer of music-led virtual realities draw on a variety of disciplines to meet the expanded needs of their compositions. One of the central disciplines to this new paradigm is humancentered design. Jerald quotes Norman in his definition of human-centered design as "a design philosophy that puts human needs, capabilities, and behavior first, then designs to accommodate those needs, capabilities, and ways of behaving" (2016, p. 2). Norman's philosophy was originally intended for product and industrial designers, but the principles are translatable. When an industrial designer is designing a door it requires the same affordances as a door designed in a virtual environment; if it is designed well, a user can understand its function and how to use it just by the way it has been designed and based on their understanding of how objects function in their experience of the physical world. Human-centered design is an important guiding force for the composer of music-led virtual realities. The user isn't asked to simply listen to the music, but actively participate in it and therefore the design of the system, and by way of it, the virtual world, must accommodate the user in their interactions. 
If how the audience interacts with the world in a music-led virtual reality is a major factor in their experience of the music, the world itself is just as important. It is here that I return to acoustic ecology and Shafer's original charge. Acoustic ecology positions the world as an audiofirst reality; it seeks to examine the relationship between humans and their environment using sound as a mediating factor. This is the goal of music-led virtual realities: to position music as the mediating factor between users and the virtual environment; to express that, regardless of whether there are visual elements or not, the music is the environment and you are present and immersed within it and granted the agency to participate in its development.

Central to this research is my music-led virtual reality, The Water Is Always Running (Buckley 2020). This work presents an unusual music-making environment, a 3D kitchen with dishwashing simulation, to explore how a defamiliarized approach to musical interaction and participation can heighten the awareness of process for the user. To explore this work, I ask the research questions:

How can a Fluxus approach to participatory music composition leverage environmental metaphors and affordances? Can a defamiliarized approach to musical interaction heighten the user's awareness of their interactions? How can a meaningful and participatory musical interaction balance between functional and creative user experiences?

To address these research questions, this thesis will begin with a review of contemporary research into audio-first and music-led virtual realities as well as sources of inspiration and reference for The Water Is Always Running in Chapter II. Chapter III will then go on to provide an overview of The Water Is Always Running and the design choices behind the project. Detailed in Chapter IV is an analysis of data collected from user interviews after experiencing The Water 
Is Always Running and Chapter V goes on to discuss key findings from the data with Chapter VI providing a conclusion. 


\section{CHAPTER II: LITERATURE REVIEW}

Since the commercial re-emergence of virtual reality in the early 2010 s, there has been a growing call in the academic community for increased research and development of audio-led virtual reality experiences ("Audio-first VR: Imagining the Future of Virtual Environments for Musical Expression,” 2018)(“FAVR 2019: Future of Audio in Virtual Reality,”2019).

Conferences focused on virtual reality and music technology have each focused on the topic with a variety of workshops and panel discussions in recent years. Starting in 2014, the Institute of Electrical and Electronics Engineers has featured special workshops in the area of sonic interactions in virtual reality with 2019's topic switching to the Future of Audio in Virtual Reality (“Sonic Interactions in Virtual Environments,” 2020) (2019). At NIME 2018, a workshop on audio-first virtual reality experiences was conducted that squarely placed the onus of responsibility for defining audio-first VR on the creators and researchers working in the area (“Audio-first VR: Imagining the Future of Virtual Environments for Musical Expression,” 2018). The press release states that "it is up to sound and music researchers to elaborate ways in which we can think natively about VR audio, and define the role of sound in the ultimate displays of the future" ("Audio-first VR: Imagining the Future of Virtual Environments for Musical Expression," 2018).

Discussing these workshops helps to outline just how accessible the concept of audio- or music-led virtual realities has become and how the groundwork for defining these experiences in light of contemporary hardware solutions is truly being laid by its practitioners. This work comes in a myriad of forms with many divergent approaches to the topic. Composers and researchers are tackling this revitalized medium with great emphasis placed on the technical aspects of spatial sonic reproduction and our perception within these spaces (Pulkki et al. 2017) (Voong \& 
Oehler 2019) (Chon \& Kim 2019) (Villegas 2015) (Cheng \& Liu 2019). Extensive work is also being done in regard to sonic interaction design for VR as well (Wozniewski et al. 2013) (Summers et al. 2015) (Serang \& Lecuyer 2013) (Men \& Bryan-Kinns 2018) (Summers \& Jesse 2017) (Johnston et al. 2008). This work is, in turn, built off of a body of literature on musical interaction design (Hunt et al. 2002) (Cook 2017) (Jorda 2004) (Machover 2002) (Schiettecatte 2004). For these reasons, in my own practice and research, I take a content-first approach and use my work as a vehicle to explore the user's perceived relationship between not only themselves and the music but also the relationship between the music and the environment.

In approaching my own practice and this research, I'm greatly interested in taking from disciplines and ideas that may not be as commonly mined for sources of inspiration in electronic music composition but are well-suited for the idiosyncrasies of virtual reality. This includes design movements such as the often industrially focused human-centered design or literary techniques such as defamiliarization. Likewise, I draw much from art movements like fluxus, in its elevation of the mundane, and from the academic discipline of acoustic ecology, the goal of which, in many ways, is to advocate for an audio-first reality. I am also drawn to recent forms of electronic music, such as vaporwave, which was gestated entirely online and is obsessed with the idea of virtual space.

I will discuss The Water Is Always Running at length in the next chapter. In this chapter, I will discuss some of the disciplines and concepts as well as contemporary research happening at the intersection of VR and music that form the foundational ideas behind The Water Is Always Running and how I have approached the creation of this music-led virtual realities. 


\section{Acoustic Ecology}

Acoustic ecology emerged from R. Murray Shafer's work at Simon Fraser University in the early 1970s. Beginning with a major sonological survey of the Vancouver soundscape, he and his colleagues created the World Soundscape Project (Wrightson 2000) in an organized effort to document the complex sonic environments that exist in both manmade and naturally occurring environments. Originally, Shafer's great concern came from what he perceived as people's inability to competently hear and understand the sounds of their environment (Wrightson 2000). Wrightson goes on to express his own personal experience with this issue as an educator. This lack of understanding can result in an entire layer of the world missed by humans. Beyond merely missing out on the vibrant soundscapes of your immediate environment, a lack of perception can also result in an inability to recognize impact. This impact is described primarily in the form of noise pollution and sonic imperialism (Wrightson 2000). What Shafer considered noise pollution and sonic imperialism is easily understood by his terms "hi-fi soundscape" and "lo-fi soundscape." Hi-fi soundscapes are environments that feature less sonic overlap and heightened perspective and spatial awareness (Schafer 1977). For Shafer, hi-fi soundscapes are often found in nature, where the sounds of animals occupy small frequency bands, the overall noise floor is quieter, and very few sounds are sustained indefinitely. This is in sharp contrast to the densely packed, industrial lo-fi soundscapes in which spatial and auditory perception are both masked by the large number of always-present sounds. It is when these dense, industrial sounds -- broadcast music and voice, traffic, etc. -- extend into unwelcomed spaces that sonic imperialism is imposed.

There are several other terms Shafer developed that are fundamental to the discipline of acoustic ecology. A number of these terms were initially laid out in Shafer's The Tuning of the 
World (1977) and all of them have been published within The Handbook for Acoustic Ecology (World Soundscape Project), which, at the time of this paper's writing, can be accessed freely online through Simon Fraser University. Many of these terms are derived from concepts common in musical analysis. This is a point worth noting as later these same concepts will be reapplied to music, however, still filtered through the lens of acoustic ecology.

Table 1

Acoustic Ecology Terms, The Handbook for Acoustic Ecology

\begin{tabular}{ll}
\hline Term & Definition \\
\hline keynotes & sounds that occur with enough frequency to be considered the \\
background against which other sounds are perceived \\
soundmark & a sound which is unique or possesses qualities which make it specially \\
sound signal & any sound which is meant to be listened to, measured or stored -- this is \\
in direct opposition to noise \\
sound event \\
a sound or sound sequence that acquires its meaning through its social \\
and environmental context \\
the smallest self-contained element of a soundscape
\end{tabular}

(Table 1, continues) 
Table 1, Continued

\begin{tabular}{|c|c|}
\hline Term & Defintion \\
\hline disappearing sound & $\begin{array}{l}\text { any class or type of sound which once existed, but has since been } \\
\text { replaced superseded, or has otherwise ceased to be heard except as a } \\
\text { museum artifact }\end{array}$ \\
\hline sacred noise & $\begin{array}{l}\text { any prodigious sound or noise which is exempt from social } \\
\text { proscription... social noises which, during certain periods of history } \\
\text { have escaped the attention of noise abatement legislators, Moozak, } \\
\text { amplified pop music }\end{array}$ \\
\hline sound romance & $\begin{array}{l}\text { any past or disappearing sound remembered nostalgically, particularly } \\
\text { when idealized or otherwise given special importance }\end{array}$ \\
\hline moozak & $\begin{array}{l}\text { generative noun to apply to all kinds of schizophonic [reproduced sound] } \\
\text { musical drool in public places, often designed to serve as a background } \\
\text { to profit }\end{array}$ \\
\hline
\end{tabular}

The terms defined in Table 1 are useful in breaking down a soundscape into its base components for analysis. In Shafer's case, that soundscape was often Vancouver, British Columbia. For the purposes of this research, the soundscape is the virtual environment of The Water Is Always Running. These terms help to categorize human perception of the soundscape. This is an important point to consider when thinking about acoustic ecology's relationship to virtual reality. These terms are bestowed upon elements of the soundscape after the fact -meaning, a soundmark or a keynote can't necessarily be 'designed in' to the soundscape. A 
sound is designated a soundmark or a keynote based solely on how the listener perceives the role of that sound within its given soundscape. When considering the overall soundscape of The Water Is Always Running, these terms will no doubt be helpful in understanding the hierarchy of the virtual soundscape as perceived by those participating in the experience.

In particular, some of the later terms listed in Table 1 are of great aesthetic concern to The Water Is Always Running. While it will be discussed in the following chapter, the overall aesthetic bent for The Water Is Always Running is decidedly nostalgic; the soundscape makes heavy use of manipulated samples from 1980s pop songs as part of the composition. The difference between sound romance and moozak is entirely personal and will be dependent upon the user's perception. This will affect not only their perception of the overall soundscape but will influence their feelings of the experience as a whole. These terms provide a way of quantifying a highly personal, but significant, part of the soundscape of The Water Is Always Running.

\section{Vaporwave}

The Water Is Always Running takes great inspiration from the internet music genre of vaporwave. This music genre and its broader recognized aesthetic is still difficult to pin beneath a single definition despite its growing status as a legitimate electronic music genre and the small, but growing body of scholarship on the topic. This difficulty is in no small part due to the tendency of vaporwave to be a sonic art concerned with cultural documentation and critique in the form of appropriation and collage. Inevitably, these definitions can sometimes include a list. Padraic Killeen, in his definition of the genre includes “vintage pop, rock, and R'n'B of the 1980s period, adverts and infomercial clips, corporate mood music, and antiquated video game sonics" all as material that is often sampled within vaporwave (2018, p. 629). Laura Glitsos, in 
her article Vaporwave, or music optimised for abandoned malls, succinctly condenses that list, noting that the genre patches together generic forms of mass media (2017). She goes on to reference Adam Trainer's assertions of vaporwave as a medium informed by oversaturation and the merging of collective and personal memories (2016).

While it is valuable to define the characteristics of the vaporwave sound, to what ends compelled these creators to begin producing this music in the first place is also a defining factor of the genre. In an interview with Private Suite Magazine, Vaporwave producer 회사AUTO described the origins of Vaporwave as "a postmodern Marxist deconstructionist critique" (2019, p. 52). While the genre has greatly expanded outward from 회사AUTO's accurate description of its origins, the overall aesthetic of Vaporwave is still incredibly derivative of the genre's initial ideals. In the first issue of Private Suite Magazine, published in June 2018, notes that "much of vaporwave is apolitical, jocular, or meme-centric" but quickly acquiesces to the genre's "strange obsession with all things economic" (2018, p. 1). The sonic palette of vaporwave is still defined by the sounds of 1980s and 1990s consumer culture. Even non-sampled vaporwave music -- one of the more recent developments in the scene -- is largely referential to these sounds.

The history of vaporwave is difficult to trace as it is a form of music that was gestated entirely online as opposed to regionally or locally. However, there are three albums that point towards the genre's development and realization. Daniel Lopatin, an electronic musician better known as Oneohtrix Point Never, released Eccojams Vol. 1 under the pseudonym Chuck Person on August 8, 2010 as a limited-edition cassette through The Curatorial Club (Person, 2010). The album's name is derivative of the music, which Lopatin has termed as "echo jams" (Person, 2010). This music, consisting of pitched down 80 s pop music drenched in reverb and delay, marked one of the earliest examples of the aesthetic choices that would define vaporwave. 
Released the following year on October 25, 2011, Far Side Virtual by James Ferraro developed the conceptual framework that undergirds the vaporwave aesthetic (Ferraro, 2020). The album handles themes such as hyperreality, the digital age, consumer culture, elevator music, and commodification. The third seminal album in the gestation of vaporwave synthesized the sound of Eccojams Vol 1 with the themes and aesthetic of Far Side Virtual to create what is considered by many to be the blueprint of the genre. Floral Shoppe by Macintosh Plus is an album by electronic musician Vektroid was released on December 9, 2011 (Macintosh Plus, 2011) and has been referred to as "vaporwave's national anthem" (Neely, 2016).

Vaporwave is music exploring the hyperreal and, in that regard, is a fitting compositional aesthetic for a piece of virtual reality music, like The Water Is Always Running. First put forth by French philosopher Jean Baudrillard (1994), hyperreality is a condition in which reality and simulations of reality are blended as such to make one indistinguishable from the other. The music is often a single sample, sourced from any number of other music genres, slowed down and looped. It renders an entire musical experience out of a single idea by zooming in on a particular moment and forcing the user to experience it over and over again, as the producer intends, until it takes on an entirely new meaning devoid from its original context. In many ways, virtual reality is the spatial equivalent: it's environments, divorced from, though referential to, reality, that are interacted with in the same ways that people interact with the physical world, but are entirely isolated from everything else and are given their own meaning as a result.

\section{Fluxus, Defamiliarization, and Human-Centered Design}

While virtual reality may consist of environments of frozen hyperreality totally devoid of context beyond any that is self-provided, those environments are built with the sole purpose of 
human occupation and must be designed to accommodate that fact. Taking this into consideration, it is worthwhile to explore human-centered design. In one of the seminal books on the topic of human-centered design, The Design of Everyday Things, Don Norman calls it a "philosophy based on the needs and interests of users" (1988, p. 188). Or, as he plainly puts it: "make sure that (1) the user can figure out what to do and (2) the user can tell what is going on" (Norman 1988, p. 188). Beyond being an immersive medium, virtual reality is also a participatory medium and in designing The Water Is Always Running, I took great care to heed Norman's advice, the implementation of which will be discussed in Chapter III.

Human-centered design was developed with the physical world and traditional digital interfaces in mind. While it unquestionably translates to virtual reality, it is worth exploring how it can be supplemented in ways that encourage the more fantastical elements and hyperreal nature of the medium when considering on overall design strategy. Virtual reality is a medium that is reality made-strange and, in this regard, it is worth examining virtual reality as a defamiliarized version of the physical world. Defamiliarization is a literary device that takes something which seems natural and unquestionable and compels the reader to questions their automated perceptions of this familiarity (Shklovsky, 1917) This is particularly fitting in the case of The Water Is Always Running as it is, in essence, the act of dishwashing defamiliarized. The whole activity is made strange so as to invite the user to question their automated perceptions of dish washing, much in the same way that vaporwave is music made strange in an effort to invite the listener to question the role of music in their culture, economy, and memories.

In asking the user to defamiliarize the act of dishwashing by way of participation, parallels to Fluxus art -- and in particular the event score -- come to mind. Fluxus was an international art and music movement and community that emerged in the 1960s that focused on 
the supremacy of the artistic process (Auslander). Fluxus artist George Maciunas described it well in a 1964 statement: "To establish the artist's nonprofessional status in society, he must demonstrate the artist's dispensability and inclusiveness, he must demonstrate the self-sufficiency of the audience, he must demonstrate that anything can be art and anyone can do it" (Auslander). To place the focus on process, an emphasis was placed on participation at every level. As Fluxus artist Alison Knowles put it, "In Fluxus, there are no actors, just simple people trying to turn you on to actions you do every day" (The University of Chicago 2012). In many ways, virtual reality experiences are like a work of Fluxus art: while there is great care taken in the creation of the virtual environment, the actual experience itself is the user's participation in the space. In the case of The Water Is Always Running, the central focus of the experience, the music, requires the participation of the user. That participation is obtained by way of washing dishes. This act of washing dishes is demonstrative of Maciunas's notion that anything can be art and anyone can create it.

In fact, the act of dishwashing and the kitchen itself has been a part of artistic discourse for decades present in seminal works of feminist art and maintenance art alike. In the video Semiotics of the Kitchen, Martha Rosler defamiliarizes the kitchen, and the stereotypical role of the housewife, by presenting utensils to the viewer and 'replaces the domesticated 'meaning' of tools with a lexicon of rage and frustration" (Electronic Arts Intermix 2006). Likewise, the act of cleaning, such as washing dishes, is present in the work of maintenance artist Mierle Laderman Ukeles (Liss 2009). For Ukeles, service acts such as cleaning are acts of maintenance as creative strategy. In creating utterly inclusive artworks, space is held for utterly inclusive meaning and interpretation. In creating The Water Is Always Running, and in the hopes of honoring the 
important works of feminist and maintenance art that it is indebted to, I aimed to design an experience that in itself could offer a wide variety of meanings and interpretations for the user.

The works of both Rosler and Ukeles also touch upon the idea of agency within a space. In particular, Semiotics of the Kitchen is layered with questions about agency within the kitchen. While the kitchen is stereotypically the domain of the woman, that indication, at least culturally, is assigned to a woman, robbing them of their agency. In Rosler's work, her act of redefining the meaning of the tools within the kitchen is a reclamation of agency. While the whole exercise feels visceral, it is also reflective. There are layers of consideration within how Rosler approaches the kitchen in the video. In circling back to human-centered design, this is something that Norman touches upon in The Design of Everyday Things. He discusses that personal reflection includes emotions such as pride, shame and guilt but also that it is the way in which an individual assigns agency to themselves and understands cause (1988).

\section{Virtual Reality Musical Instruments and Visual Design for Computer Music}

Much of what has been mentioned above is being explored in concurrent research on the intersection of virtual reality and music. In 2016, Ge Wang published a paper, entitled Some Principles of Visual Design for Computer Music, that moves many of the ideas around humancentered design closer to a music-focused taxonomy. Much in the way that has been outlined thus far in this document, Wang also divides his list of suggested principles primarily into those concerning user-centered design and aesthetics with salient points such as "do not be afraid to introduce arbitrary constraints" and "aesthetic: have one, never be satisfied with "functional"" (Wang 2016, p. 14). These principles were not designed specifically with spatial interfaces or environments in mind but their application is universal. 
Building directly off of Wang's Some Principles of Visual Design for Computer Music is the research being published on virtual reality musical instruments (Serafin et al. 2016). That work, in turn, is building off of earlier experiments conducted in VRMIs by Maki-Patola et al. (2005. VRMIs, as the authors call them, are, put simply, spatialized software instruments like those commonly found in digital audio workstations. In outlining their principles, the authors accommodate for the unique nature of virtual reality and, while The Water Is Always Running is not an instrument, there is valuable insight to be drawn from them. They note the importance of designing for feedback and mapping which is a consideration prioritized by many researchers working in this space (Paine 2015) (Hamilton 2014) but the authors also point out not so obvious considerations, such as make use of existing skills (Serafin et al. 2016). This point in particular resonates with how musical interaction is being approached in The Water Is Always Running. Instead of trying to create some new method of interaction, The Water Is Always Running leverages the common skill of dishwashing in the hopes of creating an intuitive musical interaction.

\section{Metaphors, the Virtual Environment, and Embodiment}

In Metaphors We Live By, George Lakoff and Mark Johnson (1980) write that “our concepts structure what we perceive, how we get around in the world, and how we relate to other people" (p. 124). In their view, metaphors underpin the concepts that structure everyday life and, as a result human perception of reality. Lakoff and Johnson (1980) go on to write:

The idea that metaphor is just a matter of language and can at best only describe reality stems from the view that what is real is wholly external to, and independent of, how human beings conceptualize the world -- as if the study of reality were just the study of 
the physical world. Such a view of reality -- so-called objective reality -- leaves out human aspects of reality, in particular the real perceptions, conceptualizations, motivations, and actions that constitute most of what we experience. (p. 132)

This statement echoes Maurice Merleau-Ponty's (2012) ideas around the phenomenology of embodiment, human perception, and the idea that individuals are simultaneously subject and object of the world around them. A person's perception of the world is inextricably linked to their place within that world and how they view themselves.

Lakoff and Johnson (1980) continue to discuss how human realities are tied to cultures and in turn those cultures are tied to physical environments. The ramifications of this notion is significant for virtual reality. It is impossible for humans to divorce their understanding of how to interact within a given space from their experiences within the physical world. As a result, when experiencing a virtual reality, a person's conceptualization of the virtual world is inextricably linked to their conceptualization of the physical world -- they will continuously draw environmental metaphors to the physical world they're accustomed to experiencing.

If a person's perception of the world is linked to their sense of embodiment within the world, then leveraging environmental metaphors in virtual realities increases an individual's understanding of their relationship to the virtual environment. If aspects of the virtual environment reflect qualities of the physical environment, then the user can rely on the subjectobject relationship they have established with the virtual environment's physical counterpart to gain understanding of the affordances of the virtual environment and their agency within it.

The use of metaphors as a way of understanding affordances within a virtual environment references back to Norman's thoughts on reflection as a way of understanding agency. An individual reflects upon the environment within which they find themselves and through that 
reflection, using their own metaphorical and perceptual models, they begin to understand their own agency within a space. Many works of virtual reality that proved to be a point of inspiration for this project -- alongside those already stated by Rosler, Ukeles, Brecht, and others - make use of user reflection and metaphors to help the user understand their own agency. In the case of Rob Hamilton's Coretet (2018), the composer has created virtual instruments that are metaphorically reminiscent to a string quartet to help the user understand their musical functions. An another work of Hamilton's, ECHO::Canyon (2013), the composer attaches musical functions to the physiology of a flying creature and allows the user to fly around a virtual landscape. This work relies on the user's perceptual model of a spatial environment and the metaphor of a canyon as a vehicle for exploring musical content. 


\section{CHAPTER III: THE WATER IS ALWAYS RUNNING}

\section{Project Description}

The Water Is Always Running is a music-led virtual reality consisting of a 3D kitchen with dishwashing simulation that highlights a defamiliarized approach to musical interaction (Figure 1). The aim of this piece is to highlight awareness of process in the user by taking a simple and relatively universal act, namely washing dishes, and transforming it into an act of musical participation and discovery through defamiliarizing the process.

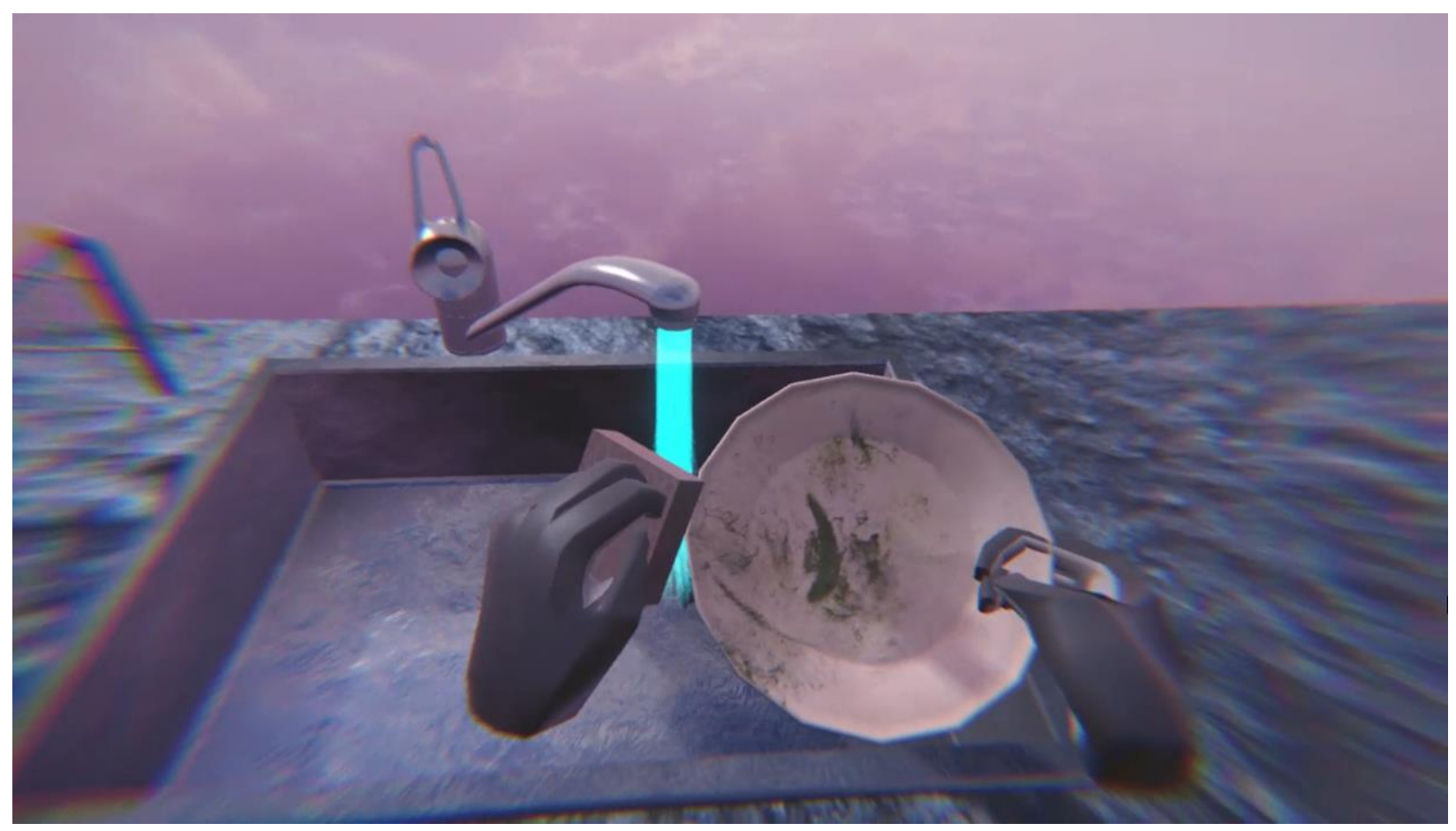

Figure 1. User view of The Water Is Always Running

This project was developed using the Unity game engine (Unity Technologies 2020) to develop the virtual environment, Ableton Live (Ableton 2020) to compose and produce the music, and Audiokinetic Wwise (Audiokinetic 2020) to implement the audio. Development was 
also conducted using an Oculus Rift S. Finally, central to the development of The Water Is Always Running was the Leap Motion Controller (UltraLeap 2020). This hand-tracking module allows users to interface with digital environments using just their hands by tracking real world gestures and movements and mimicking them using an in-world virtual pair of hands (Figure 2). This was implemented into Unity using the Orion Hand Tracking software and Leap Motion SDK and employed prefabricated interaction components. I wanted to minimize user friction with the interface and being able to bypass the use of any controllers would, hopefully, altogether eliminate the need to supply the user with an in-world tutorial and instead place a greater emphasis on environmental affordances and discoverability.

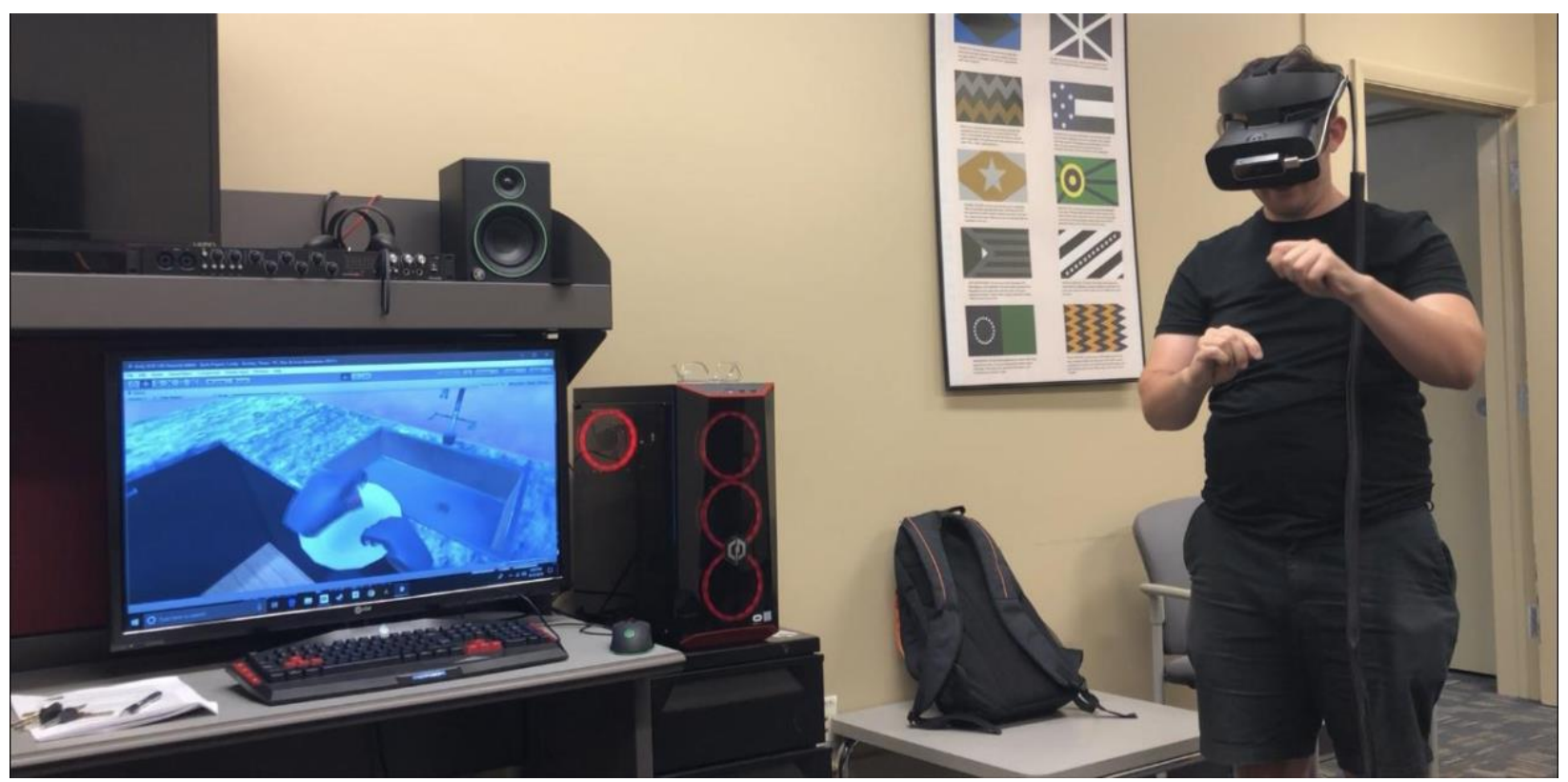

Figure 2. Early development image showing use of Leap Motion Controller

The virtual environment that comprises The Water Is Always Running consists of a floating platform modeled after a typical American kitchen (Figure 3). The player is placed in front of a long row of granite countertop with a single compartment sink in the middle. To the 
left of the sink is a drying rack and just a little further beyond that is a three-tiered shelf.

Scattered about the shelf and countertops are eight dishes, four bowls and four plates, and a sponge. Beyond the platform comprising the kitchen is a skybox trapped in a permanent pink and blue sunset. At the beginning of the experience everything begins still and silent except for the sight and sound of the running water coming out of the faucet. Should users desire to turn the water off, they will be unable to. Sonically, it acts as the keynote to the whole soundscape. The faucet handle is interactable, however, it begins to spin when touched, completely divorced from gravity. The faucet can't be turned off and the water is always running.

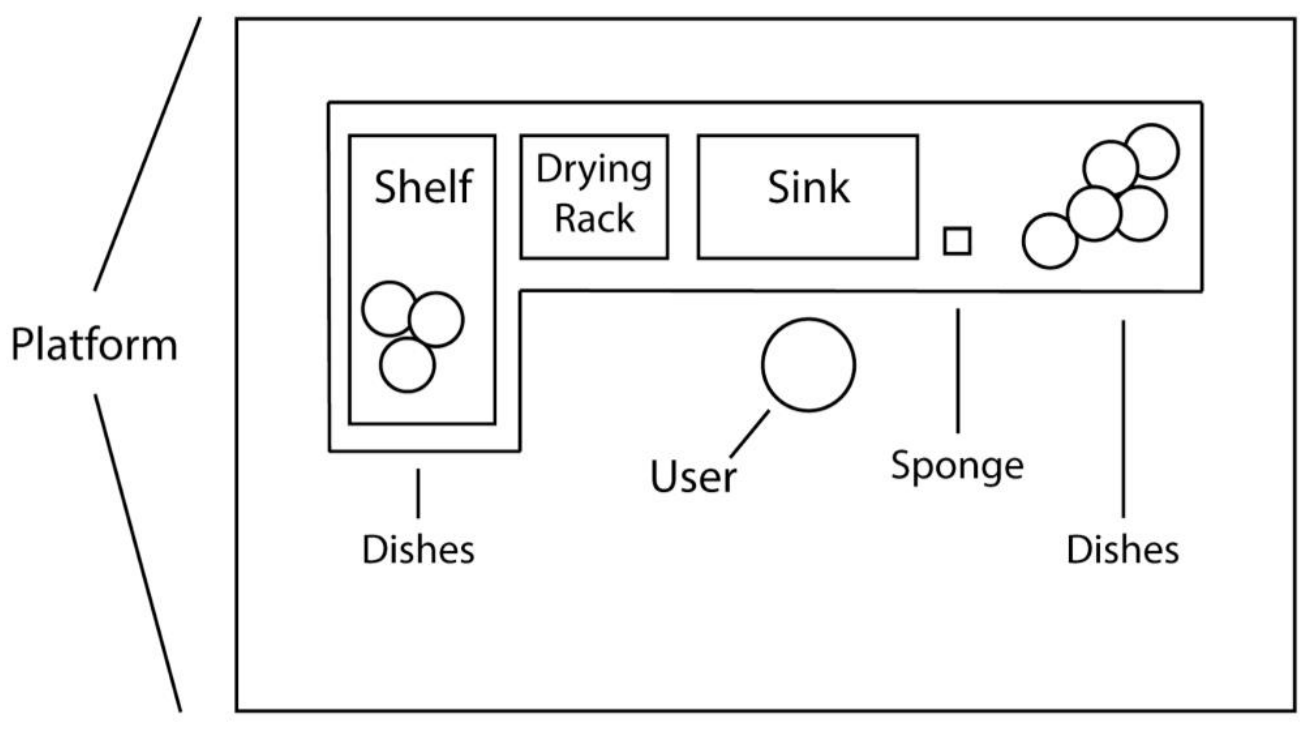

Figure 3. Layout of The Water Is Always Running

Each dish has two visual states - dirty and clean. At the start of the experience, every dish is scattered about the shelves and counter, dirty. The dishes can be shifted from dirty to clean by one of two methods: scrubbing with the sponge or running the dish under water. Once a clean dish is touched again, however, it becomes dirty once more. When a dish is touched, a single, 
bell-like musical note sounds and a four measure musical phrase begins looping. This loop has four states that correspond to actions taken by the user. Initially, the music begins in its "dirty" state that corresponds to the dirty visual state of the dish. When a dish is cleaned - the music transitions to its clean state. Whenever a dish is held under the running water, the musical loop transitions to a third, "rinsing" state. This state is only active whenever a dish is under the faucet and once removed, transitions to the cleaned state.

This loop continues until the dish is placed on the dishrack, at which point it transitions to an ending loop that does not repeat once it reaches its conclusion. If the player touches the dish again, though, it returns both visibly and sonically to its dirty state. If a dish or the sponge is thrown off of the platform or onto the ground, it will re-instantiate above the counter. While each dishes' corresponding musical loop is four bars and can transition back and forth between the same designated states, each dish corresponds to a unique musical voice. Four of the dishes have distinct loops made from manipulating isolated vocal tracks from 80 s pop songs. The remaining four each comprise of drums, synth bass, arpeggiating electric piano, and a synth pad generated from sample instruments in Ableton Live. The sponge has a single shaker percussion loop that is triggered on or off depending on whether the user is holding it or not. Finally, playback speed for each dish is mapped to the $\mathrm{Y}$ axis. If a dish is lifted higher, it's playback speed will increase and, as a dish is lowered, it's playback speed will decrease.

\section{Design Process}

\section{Interaction Considerations}

I wanted to create a music-led virtual reality that explored the idea of an embodied musical composition that was meant to be experienced by way of participation, test it with users, 
and see if I could establish a blueprint for future works. My early work on this project explored research on virtual environments that supported the composing process, which prompted me to explore the development of a framework for composers specifically in virtual spaces (Buckley and Carlson 2019). I wanted to develop best practices for composer's looking to make 3D virtual environments that exist as primarily embodied musical installations where the user can interact with and have agency over this space. The Water Is Always Running began as a vehicle for me to test those ideas out.

This work focused heavily on discoverability and affordances. In considering an effective environmental metaphor that would translate well to this idea of an embodied musical installation, I ultimately settled on an American kitchen and more specifically the act of washing dishes as the foundation for the musical interactions in The Water Is Always Running.

Foundational in the early iterative stages of The Water Is Always Running was the design theory of human-centered design, which Don Norman describes as "a philosophy based on the needs and interests of the user, with an emphasis on making products usable and understandable" (Norman 1988, 188). A successful music-led virtual reality that is accessible to many should be tutorial-free, in order to create a virtual world where the musical interactions feel as intuitive and understandable as a well-designed object in the physical world. The use of a well-designed tool or piece of furniture can be discovered and understood just by the way it is designed. My focus on discoverability and affordances led me to settle on two foundational design choices: to forgo using any sort of in-hand controller and to lean heavily into a physical-world environmental metaphor.

The first choice, forgoing any sort of in-hand controller, was achievable through the use of a Leap Motion Controller. This hand-tracking module attaches to the front of a VR headset 
and replicates the user's gestures and movements in virtual space. This allows for the user to interact and discover the virtual environment in the same way they would a physical environment: through sight, sound, and touch. The interactions are integrated using Leap Motion's Unity SDK -- specifically the Orion software. The Orion software provides capabilities for two interactions: pinch and grab. The SDK provides an extensive interaction manager as well as Unity prefabs for the virtual hands. Dishwashing is fundamentally an interaction of grabbing and, as a result, there was no need to make any major changes to how the interactions functioned in Unity. The system had to accommodate for the lack of haptic feedback but in a system where sound is the primary means of interaction, the lack of haptic feedback was compensated for using aural feedback.

The second choice, to lean heavily into a physical-world environment as a metaphor for how to interact within the space, ultimately proved to be a fruitful creative limitation. If using a Leap Motion Controller meant that I could employ the most fundamental human tool available, the hand, as the primary vehicle for interaction, then I wanted to rely on interactions that the hand is already used to performing in the physical world. The Leap Motion Controller excels in tracking simple interactions like pointing, pinching, and grabbing. I settled on the metaphor of dishwashing because its core interaction is grabbing and scrubbing: pick up a dish, pick up a sponge or brush, and scrub the plate with it until the plate is clean. This core dishwashing loop is easily replicable in a virtual environment. It is also an interaction that keeps the user in one place and watching their hands as they perform the act. This is also important to note as VR is a largely stationary experience and since the Leap Motion Controller is mounted to the front of the headset, it keeps the users hand in sight of the sensor in the controller. And, by employing an 
interaction that requires two objects to come in contact with each other, collision detection can be used to trigger an output.

Dishwashing also has a clear set of inputs and outputs that translate well for use in a virtual environment when streamlined to accommodate for the limitations of the medium. When washing dishes in the physical world, there are a number of factors that the user has to consider: water pressure, water temperature, the amount of force being applied to the dish with the sponge, the amount of buildup on the dish, and the length of time spent scrubbing are just a few nuanced factors in a seemingly simple act. Because many of those parameters just listed require extensive touch and pressure, the interaction has to be simplified to translate well to virtual reality. What does translate well is the core physical act of grabbing two objects and bringing them together and the strong visual difference of a clean dish from a dirty dish.

Choosing to use the environmental metaphor of a kitchen and the act of washing dishes meant that if the experience as not properly subverted the user would, ultimately, just feel as if they were merely washing dishes. To remedy this, I leaned heavily into making the act of dishwashing a defamiliarized one in the same fashion as one might find a common activity subverted in a Fluxus event score. For example, in George Brecht's 1959 score for Drip Music, the performer is instructed to take an empty vessel and place it under a source of dripping water (Friedman et al. 2002). In the Fluxus tradition, an event score such as Drip Music is meant to highlight this simple act as the totality of artistic expression. Some event scores, like Ken Friedman's Fruit Sonata, in which the performer plays baseball with a fruit, takes an activity that is well known to the performer and audience and subverts it (Friedman et al. 2002). In The Water Is Always Running, the user is the artist-performer of a virtual Fluxus event. However, it is the affordances present in the environment that serve as the score. When a user enters into the virtual 
world of The Water Is Always Running there is nothing that they can interact with other than the dishes and the sponge. Having only the sponge and dishes to interact with is environmentally similar to Fruit Sonata only instructing the performer to play baseball with fruit: the power and the beauty of that performance is how the performer chooses to interpret the instructions. The point of interest with The Water Is Always Running is the same: what will the user do with sponge and the dishes?

\section{Musical Considerations}

What does it mean to create a "musical world" in virtual reality? Acoustic ecology posits the primacy of the sonic experience in the perception of individuals. It advocates for a world in which sound as a human sense is of the highest consideration. This desire to elevate sound, while the main focus of acoustic ecology, is an uphill battle that Schafer himself was well aware of. In the introduction to The Tuning of the World, he writes that "noise pollution is a world problem" and that it occurs when "man does not listen carefully" (1977, p. 4). That his ultimate diagnosis is that the planet is rife with noise pollution speaks to his assessment of humanity's capacity to listen.

I am drawn to working in VR because I can design the virtual world as I see fit and create VR experiences that are centered around their musical content. In VR, the primacy of sound no longer needs to be a long-dreamed of fantasy in which the aural experience becomes humanity's primary concern. Instead, games and experiences, like The Water Is Always Running, can be designed to create pockets of multimodal reality in which the user can be led to focus on senses and experiences at the creator's discretion. However, in the planning and development for The Water Is Always Running, it quickly became apparent that it is one thing to say a VR experience 
is music-led and it is another thing entirely for the user to feel the same way. VR, in many ways, transcends normal conversations about artistic truth in authorial intent. If a reader disagrees with an author's interpretation of their own novel, it does not stop the reader from experiencing the medium as it is intended. Thematic disagreements do not stop the reader from reading the book. In the case of interactive experiences that require participation from the user, if the creator introduces an element of an interaction that they believe is invaluable but is not understandable or achievable to the user, then the user may be unable to fully participate in the experience. This lack of understanding is fundamentally a design failure and not a failure on the part of the user.

Early in the development of The Water Is Always Running this problem of authorial intent vs user experience became readily apparent in my own design process. The central interaction, musical segments triggered by and modified through manipulating the dishes within the space, was the same in early iterations as it ultimately was in final designs. However, there were originally only four plates with each plate corresponding to two musical loops, one instrumental and one vocal (Figure 4). Like the final version, as the plates were changed from dirty to clean and back again the corresponding loops would change as well. The big difference in this version was that every loop was locked in at the same tempo and timing with all other loops. Meaning, each loop consisted of a four measure phrase and when a loop would transition to a new state, instead of starting at the beginning of that new four bar phrase, it would transition to the same place in the new four bar phrase as the loop currently playing. If, for example, a loop was transitioning from its "dirty" state at beat two of the second measure then the "clean" loop would start at beat three of the second measure as opposed to beat one of the first measure. 


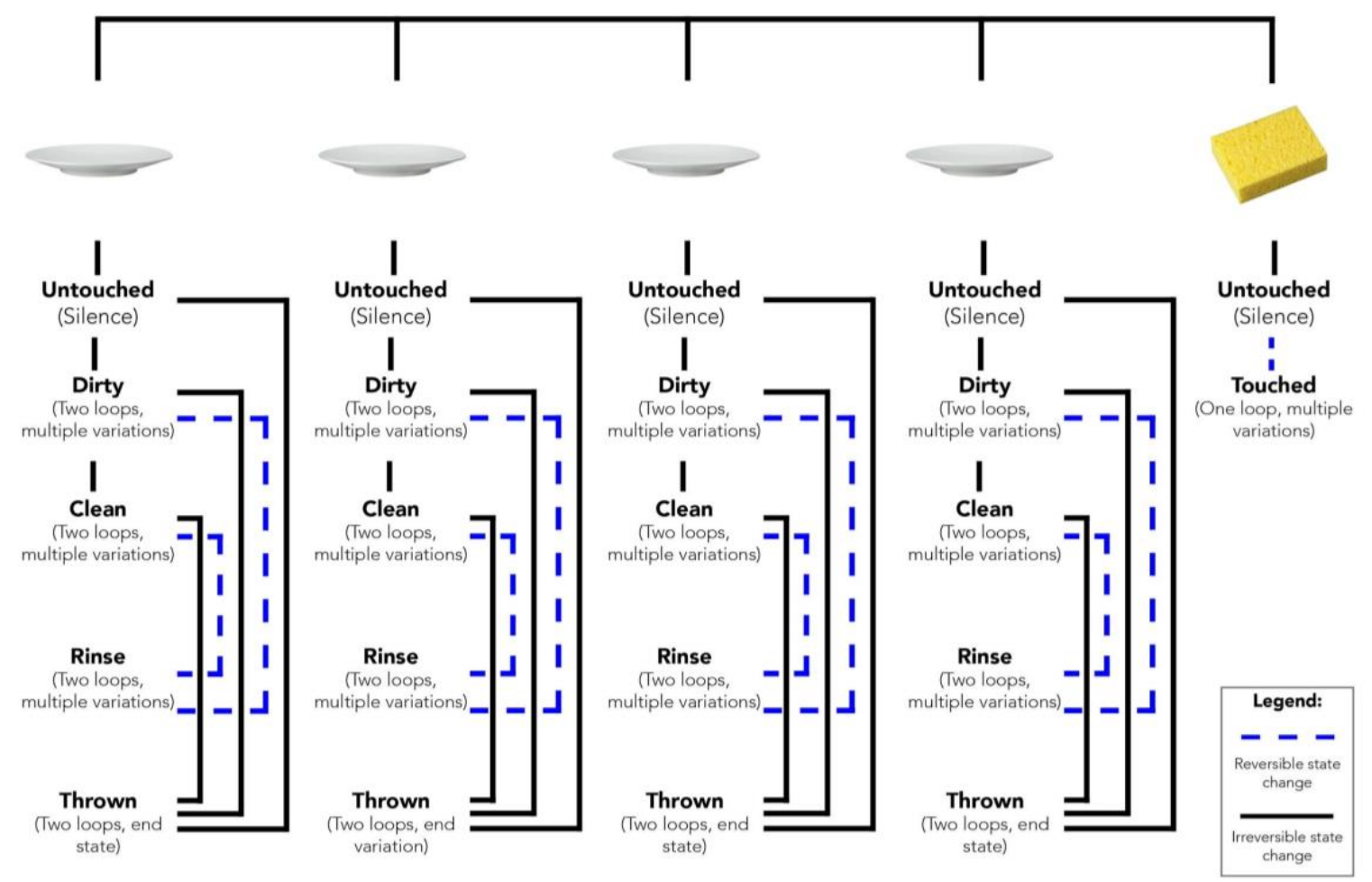

Figure 4. Early mapping schemata for musical interactions in The Water Is Always Running

The ultimate effect was one of complete musical cohesion between the plates and their various loops but perceptually lacking distinction for the user. On one hand this was musically very interesting. The resulting interlocking composition consisted of eight instrumental voices each comprising four bar loops that could be interchangeable with one another when playback was layered. If every dish was in its clean state vs its dirty state the music had a distinctly different feel, but the music could also function in many states of in-between -- two dishes dirty with six clean, one clean dish and seven dirty, etc. In pre-study testing and feedback sessions, users focused primarily on the act of dishwashing and gave little thought to the music. Most simply assumed it was a soundtrack that they had no effect over. With only four dishes the 
resulting soundscape quickly became densely layered and felt more like a soundtrack as opposed to a means of interaction.

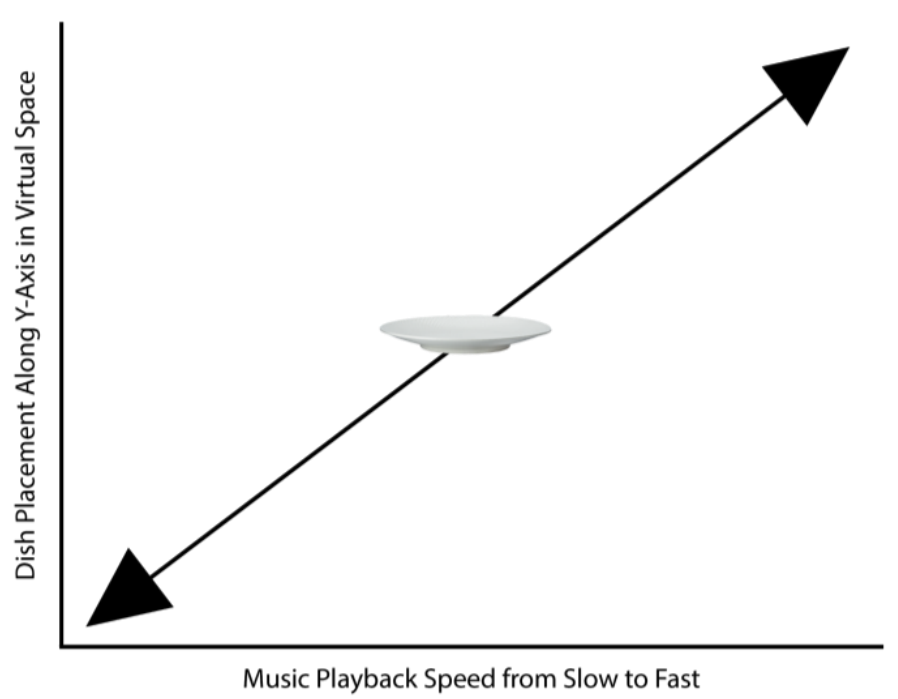

Figure 5. Visualization of music playback speed mapped to dish placement along Y-axis

It became apparent that when the dishes' corresponding loops were locked into the grid, the user did not feel fully capable with them -- lacking this important aspect of the interaction. Every change to the music was completely locked to the grid and, as a result, early feedback from colleagues who tried the software in test iterations thought that their interactions had no bearing on the music. The interaction lacked what Garth Paine has dubbed the "techno-somatic dimension," a theoretical design parameter that explains the relationship between performer and instrument as one in which the instrument is an invisible, embodied extension of the performer with which they feel totally capable and are primarily concerned with the feedback it provides (Paine 2015). In a musical virtual world this relationship is blown up to the scale of the entire environment and, in the case of The Water Is Always Running, the user and the dishes. 
To increase system feedback and discoverability for the user, the musical output of the interactions was defamiliarized even further. Instead of each loop being locked in at the same tempo, playback speed for each loop is now mapped to the Y-axis of its corresponding dish in 3D space (Figure 5). This means that as a dish is lifted higher in 3D space the playback speed of the loop increases which results in a faster tempo and higher pitch. The reverse is also true; as height decreases, playback speed slows down. Furthermore, instead of two musical voices -- one instrumental and the other vocal -- corresponding to a single plate, the final design had the number of dishes doubled from four to eight with each voice corresponding to its own dish (Figure 6). The resulting musical output is collagist in nature. However, while the end result could be a perceived diminished relationship between the loops collectively, the relationship between the loops and their corresponding dishes is perceivably strengthened as the lack of collective musical cohesion means that individual parameter changes can stand out more easily.

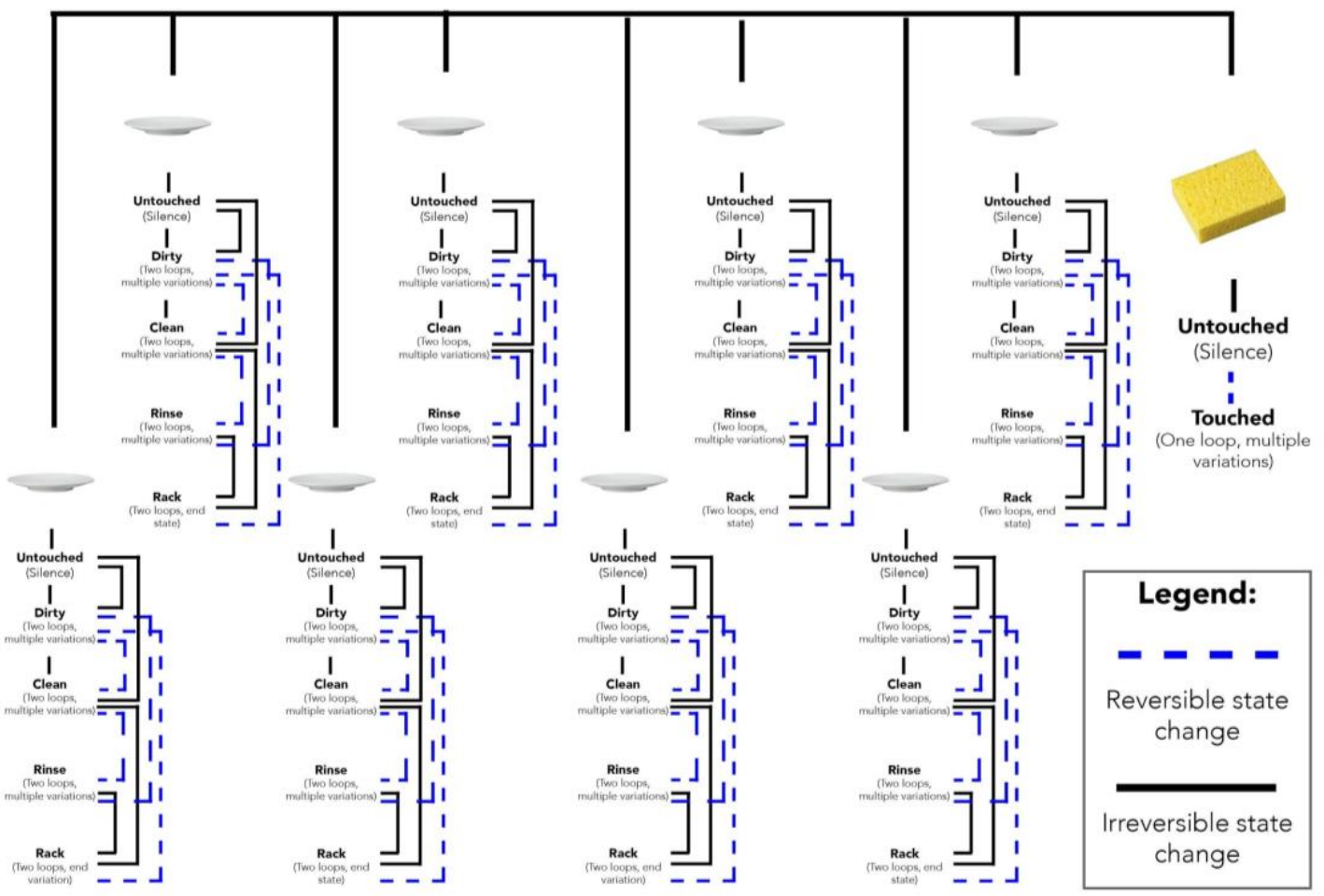

Figure 6. Final mapping schemata for musical interactions in The Water Is Always Running 


\section{Environmental Considerations}

The 3D environment for The Water Is Always Running was designed first and foremost in service of the user interactions. In order to use a dishwashing metaphor, real life objects become necessary. Placed within the virtual kitchen environment are three fundamental objects that comprise a dishwashing experience: the sink, dirty dishes, and a sponge. The rest of the environment is designed to reinforce this mechanic to the user and point them back to the sink. Instead of placing the kitchen in a room within a house, I chose to place it on a floating platform surrounded by the skybox to create an environment that is unusual and with little distraction. I chose to forgo the typical kitchen environment to not only reinforce the absurdist nature of the experience but to also make it clear to the user that there is nothing else for them to do. The implication of four walls, door, and possibly windows implies that there is something beyond those four walls. I wanted to show the user immediately that there was nothing else available for them. Originally, the platform was placed in an entirely darkened space with a single spotlight on the kitchen, but it created an overall oppressive environment that implied a more sinister experience than intended. Switching from a near black to an entirely white environment created the same effect. Placing the entire experience in a sunset skybox proved the same effect, showing to the user that there was nothing else in this world for them to do, while removing any sense of threat that early pre-study participants expressed in the black and white variations.

Leaving the sink faucet running at all times was also done intentionally to reinforce where the user needed to place their attention. Upon entering the virtual environment, the only sound the user hears is that of the faucet. They are also placed right in front of it. The user can do whatever they want in the space, however, there is always the tension present from the running water that they can't turn off. It's a sonic reminder of the purpose of the experience; the water is 
running so the only agency the user has is to wash dishes. This design choice leverages the user's understanding of the metaphorical and perceptual models present within the space to inform their agency and influence their perception of the power they have within this space. This same effect is employed in an opposite fashion to compel the user away from dishwashing by making it difficult to complete the task through making clean dishes dirty when picked up. This robs the user of a certain level of expected control as a way of prompting the user to think the differently about their perceived expectations for the space.

The faucet also serves as a complex role in the soundscape of this particular virtual world. In an acoustic ecology analysis of The Water Is Always Running, the faucet serves at the keynote and, depending upon the user's personal experience, the soundmark for the environment. The unrelenting sound of the running water provides enough contrast to the quick and immediate chime that plays when the user picks up a plate as to thus denote that chime as a sound signal, something different and worth paying attention to. This relationship is emphasized further when the loops, the significant sound events of the environment, begin to layer into the soundscape and the overall density increases, changing the soundscape from being a hi-fi soundscape with little overlap and increased spatial understanding to being a lo-fi soundscape, where the overlap in sound is far greater and sonic spatial awareness can be diminished.

The two other objects in the space, the three-tiered shelf and the drying rack, serve different purposes to help the user grasp available interactions. The shelf serves as a starting point for a number of dishes to increase discoverability of the relationship between the $\mathrm{Y}$-axis and loop playback speed. It forces the player to interact with the dishes at a height beyond what would necessarily constitute normal for dishwashing. The drying rack is the stop function for the music loops, leaning into the normal function of a drying rack as an end point for the act of 
dishwashing. The close proximity of the drying rack to the sink is meant to influence user choice over where to place dishes when they are finished with them as opposed to placing clean dishes on the farther away shelf.

\section{Supporting Design Through Conceptual Vision}

The central concept of The Water Is Always Running is overconsumption. This concept was developed as a way to reinforce and explore the kitchen and dishwashing metaphor used as the foundation for user interactions. The primary function of this work was to explore the relationship between user, interaction, and musical process, so it was acceptable to have aesthetic choices emerge in support of design choices. While this could have resulted in a subservience to the interaction design, the synergy created between the two user interactions, the seemingly never-ending repetition of the dishwashing, and the project aesthetics help greatly to reinforce the concept.

The impetus for the theme of overconsumption was born from the notion that dish washing is one of the more prominent ways in which any individual is directly responsible for the byproduct of their own consumption. In American culture, the norm is to use something and then throw it away (or recycle or compost for those privileged to be able to do so), at which point then that object is somebody else's problem entirely. Whereas in many American homes, a dish is a vessel for consumption that must be cleaned before it can be used again. There is a certain level of personal responsibility there that is not present in the relationship between person and garbage can.

In thinking about this notion of overconsumption, I found myself drawn to vaporwave as the thematic reference point for the music. The genre's somewhat stereotypical characteristics of 
off-kilter loops and a tendency for pitch and tempo to vary unevenly in a single piece of music found a perfect home in the musical interactions designed for the dishes because the fluctuating playback speed results in loops that feel more and more unsteady as the user interacts with a particular dish. Often to achieve this effect, vaporwave music is heavily edited and processed. For the music of The Water Is Always Running, instead of heavily processing it using software to achieve the desired aural qualities, I chose to rely on the combination of the changing playback speed as a result of the dishes moving along the y axis in the virtual space and the sonic density that comes from multiple plates playing at once.

Vaporwave, as a genre, places a heavy emphasis on interpreting consumer culture -particularly the culture of the 1980 s and 1990 s -- as an act of collective nostalgia for things that may never have happened. It's a way of misremembering and reliving a past that could've been as opposed to was -- often in a much more positive, commercialized light than was true. In this way, there is a connection between the dirty dish and the vaporwave aesthetic of its corresponding music: a dirty dish is, in a sense, a document of the past; a testament to that which was once consumed. However, as a dish is washed and used over and over again, any memory of a meal or particular moment associated with it becomes inevitably warped and diluted by all the other moments and meals shared on that plate. In The Water Is Always Running, newly composed music is merged with warped snippets of 80s pop songs and flattened into an entirely new dynamic that marries my memories of these songs as a listener with my interpretation of them as a composer. And in the hyperreality that is the virtual environment this reinterpretation of the music is validated over and over again by the user's actions. This hyper-compressed portrait of a musical experience is played out in the same way with the dishes. The user cleans the dish but touching it again makes it dirty once more. Throwing the dish away only brings it 
right back. The process of creating and consuming a meal is forgone and compressed into just the act of touching the dish and, in it, the physical experience of cleaning the dish becomes the primary method of experiencing the dish with all context to be garnered and drawn from the musical experience that takes place. 


\section{CHAPTER IV: STUDY AND DATA ANALYSIS}

After development of The Water Is Always Running, I conducted a study with six participants to explore their feelings and perceptions about the experience. Each participant's session lasted approximately a half hour. The target demographic for the study was members of the university community. This demographic was chosen for their accessibility but also because Illinois State University, as a whole, has a demographic makeup similar to the state of Illinois as a whole (2019). The participants were gathered through personal contacts. There were four male and two female participants with ages ranging from early 20 s to early 40 s. Each participant regularly washes dishes in their everyday life though not every participant had experience with virtual reality before the study. The open-ended interview questions were analyzed for relevant themes. To explore this work, I ask the research questions: How can a Fluxus approach to participatory music composition leverage environmental metaphors and affordances? Can a defamiliarized approach to musical interaction heighten the user's awareness of their interactions? How can a meaningful and participatory musical interaction balance between functional and creative user experiences?

\section{Study Design}

Each participant took part in a half hour session that consisted of a section of establishing questions, followed by an open-ended period of exploration in The Water Is Always Running, and finishing with an interview focused on the participants feelings and perception of the experience. The establishing questions shed light on participant's experience with virtual reality and the frequency with which they conduct both general domestic tasks as well as washing dishes. While there is a general universality to the process of washing dishes in America, it was 
still important to note whether the participants had any personal experience with dishwashing as this would give insight into how they approached the VR experience.

Participants were consistently new to virtual reality as a medium. Two participants had never used VR before. The remaining four had, however, three of them responded that they had used VR only once before with the fourth having used it only twice. Participant responses when asked how many times a day/week they completed domestic tasks were widely varied. One participant stated that they completed, on average, zero domestic tasks in a day and five total in an average week. On the other hand, another participant estimated doing an average of $12-15$ domestic tasks on any given day with an average of just over 100 in a given week. This particular participant made note of the fact that they complete a number of domestic tasks outside of the home as well. When asked specifically about the frequency with which they wash dishes, half of the participants quantified their dish washing as x many times a week while the other half of study participants stated that they wash dishes daily. This distinction may not hold any bearing to how skillful these participants are at washing dishes but could be indicative of personal or social habits and pressure that could influence how they interact within the virtual environment.

Table 2

Participant Responses to Establishing Questions

\begin{tabular}{llll}
\hline $\begin{array}{l}\text { Domestic tasks } \\
\text { completed in a day: }\end{array}$ & $\begin{array}{l}\text { Domestic tasks } \\
\text { completed in a week: }\end{array}$ & $\begin{array}{l}\text { Frequency of } \\
\text { dishwashing: }\end{array}$ & $\begin{array}{l}\text { Number of times } \\
\text { using virtual reality: }\end{array}$ \\
\hline 4 & 28 & four times a week & once \\
0 & 5 & once a week & once
\end{tabular}

(Table Continues) 
Table 2, Continued

\begin{tabular}{llll}
\hline $\begin{array}{l}\text { Domestic tasks } \\
\text { completed in a day: }\end{array}$ & $\begin{array}{l}\text { Domestic tasks } \\
\text { completed in a week: }\end{array}$ & $\begin{array}{l}\text { Frequency of } \\
\text { dishwashing: }\end{array}$ & $\begin{array}{l}\text { Number of times } \\
\text { using virtual reality: }\end{array}$ \\
\hline $12-15$ & 105 & every day & once \\
$4-5$ & $28-35$ & once a day & never before \\
6 & 30 & twice a day & never before \\
$2-3$ & 14 & every day & twice \\
\hline
\end{tabular}

Participants were greeted and introduced to the study with an explanation that they were going to explore a virtual environment with total freedom to conduct themselves within it however they would like. For the period of open-ended exploration in The Water is Always Running, participants were guided through how to wear the Oculus Rift S and headphones used for testing but, beyond that, given no instructions aside from how to remove the headset when they personally decided they were finished. This was done in part to see how long the experience could hold their attention, based on their own perception of the "goal" of the experience. By also not providing a list of instructions on how to participate in The Water Is Always Running, it was my intention to give the user freedom in how they approached the dishwashing metaphor. While the core of the interaction is reliant on the act of washing dishes, much of the experience's depth -- and the intention in its design and overall meaning -- is in how the user chooses to subvert the act. Furthermore, I wanted to see how users discovered the different mechanics present in the design without using a set of guided instructions to tip the scale in their favor.

The interview portion that followed focused on four areas:

- the participant's feelings about the experience 
- their expectations as they explored the experience

- their personal analysis of the experience

- a number of questions probing the metaphors and affordances of the experience

The goal of these questions was not to lead the participant to a specific conclusion, but instead to understand the effectiveness of the defamiliarized approach of the musical interaction and participation of The Water Is Always Running through the lens of their experience with it.

\section{Major Themes}

Participants gave a wide range of responses to the 15 questions asked of them (see Appendix A) and their answers can be broadly parsed in pertaining to one of five categories: environment, interaction, music, feelings, and experience. There is extensive categorical overlap within the answers, however. Unless a question specified one of these five areas -- and even then -- participants spoke to the general multimodality of the entire experience.

Consistently across the board, there were a number of specific takeaways. Every participant was able to interact with the environment without any instructions beyond how to wear the Oculus Rift S and headphones. Every participant was also able to produce sound, even though understanding of how that sound was being produced was mixed. In total, participants spent an average of 8:30 freely exploring The Water Is Always Running before they felt they were finished and took off the Rift S; the longest individual session was just over 11:00 and the shortest 5:45. 


\section{Environment}

Participants described the environment as "dreamlike, otherworldly, strange, and a fantasy" in questions asking them to describe their feelings about the experience as well as describing the experience itself (personal interview 2019). Participants attributed the dreamlike quality of the experience to the presentation of the environment with Participant 6 noting that the experience "could've just been a fake kitchen area in a house but it was elevated in that sky... the fact that I was up in the sky, that really did something for me." Most participants understood the environment to be a kitchen without any walls, floating in the sky. However, Participant 3 described the environment beyond the platform as a sky and reflective pool. Participant 3 in particular was consistently focused on the water as a central part of the whole experience noting that "the water streaming [from the faucet] was kind of like a waterfall" (personal interview 2019).

In fact, the faucet was a notable -- and often emotional -- part of the experience for many participants. For a number of participants, it held heavy sway over their emotions, interactions, and the overall experience. Participant 3in particular -- the one who likened the faucets presence to that more of a waterfall -- had a relatively complicated relationship with the faucet overall. The waterfall description seemed strictly aesthetic and meant to tie into their overall perception of the environment as one of a sky with reflecting pool, then anything else. This poetic description of the faucet as a waterfall is incongruent with how they perceived its role in their interactions, which featured language of frustration. This is something else that will be discussed when going over the collective and individual emotional responses to The Water Is Always Running. 
In some responses, participants would treat the kitchen as separate from the skybox beyond the platform, instead of thinking about the virtual environment as one cohesive space, with Participant 1 stating that it was "odd to see something common (the kitchen) in a strange environment" (personal interview 2019) Participant 5 in describing what they had experienced said, "I was in a kitchen with no walls and I was surrounded by sky and floating," reflecting that it was "weird that there's this floating room" (personal interview 2019). Instead of accepting the totality of the virtual environment as a being cohesive, participants consistently described the space common to their everyday life, the kitchen, as being separate from the fantastical elements beyond the platform that occupies the interactable space. Interestingly, participants often felt that the music was the bridge between the two seemingly different aspects of the environment -- the platform and everything beyond it. This is something that will be explored more in depth when the sound of the experience is discussed but is worth noting that, at least partially, the intention to create a Fluxus-inspired environment was successful

The space beyond the kitchen platform was one of interest to many participants. It did consistently reinforce to participants that the focus of the experience was the contents of the platform that they stood on. Participant 5 noted in their interview that, "there was nothing else to do so there's this assumption that I'm going to clean the dishes" (personal interview 2019). Participant 6, "as soon as I realized I was in the sky I wanted to go and look at the ground" (personal interview 2019). Every participant indeed did attempt to look over the edge and half of them, recognizing that they were in a virtual environment, attempted to walk off the edge. Participant 1 was afraid of heights and instead of viewing the space beyond the platform in the poetic terms the other participants used, they noted that it felt overwhelming and as if they were trapped. Only one participant was able to bring themselves to step off of the edge, though (the 
user will just step onto an invisible platform instead of entering a freefall). The inability to step off of the platform is well reflected in Participant 2's assessment of the environment as one that "felt like its own space, like it was real" in which the consequences of the real world could hold sway in this virtual world as well (personal interview 2019).

\section{Interaction}

Participants' interactions within the space were overwhelmingly informed by the dishwashing metaphor. When describing their experience, Participant 1 noted that their "natural instinct was to wash" (personal interview 2019) Some participants noted that this was because of their own sense of immersion in the virtual space. In discussing their expectations upon first entering the experience, Participant 5 said, “It felt so real. It felt so normal. So I was like, I'm just going to wash this dish and it's going to be clean and I'm going to put it away." This can be partially explained by the staging of the experience. When Unity loads up The Water Is Always Running, the user is always placed immediately in front of the sink, with the water running, and a stack of dirty dishes to to their right and the shelf with dirty dishes to their left. It's unlikely for users to be able to take in any initial spatial information that would allow them to perceive this function of the environment in any other fashion.

While the design of the environment influenced participants' interpretation of how the space could be interacted with, their own desire to explore the space held just as much influence. When each participant was asked what they wanted to do first in the experience, the answer was, near universally, “touch things.” However, even though Participant 3 didn't respond with touch things did respond with "shut that water off," or, an act of touch over concerns of the water being wasted. Participants noted that it was because they were able to see their hands and discovered 
the ability to use their hands that they wanted to touch everything, with Participant 2 saying, "I knew as soon as I saw my hands there were things around I could touch" (personal interview 2019). They continued, "The first thing I saw was my hands. The first thing I wanted to do with them was pick something up -- the dishes" (personal interview 2019). Participant 4 put it succinctly, "I wanted to touch stuff and get something to do something" (personal interview 2019).

Constraints and limitations. The virtual hands, while feeling largely intuitive to the participants, weren't without difficulty. For many people, their hands are the pair of tools with which they're the most intimate and, therefore, if the tracking doesn't feel perfect to the user, they will inevitably notice the differences -- which the participants did. After each interview, participants were asked if they had anything else they would like to discuss and Participant 2 noted that "if I tried to be too quick with my gestures, there was a latency" (personal interview 2019). Participant 5 shared that, "small things like grabbing the dishes or the sponge was hard" (personal interview 2019). These participants quickly adjusted, with Participant 6 expressing that, "I figured it out. If I moved my [real] hand slowly, [the virtual hand] would keep up with it" (personal interview 2019).

Once participants began to explore everything and gain an understanding of what could be done within the space, they began to develop different goals for what they wanted to achieve with their time in the environment. The first major goal for some participants was to clean the dishes -- "I better try to wash these dishes" as Participant 3 noted, a statement that could be indicative of personal social pressures surrounding the act of dishwashing (personal interview 2019). The impetus for this goal and reasons for its emergence have been discussed at length above. The second major goal for participants was total subversion of what they believed to be 
the "correct" way of participating in The Water Is Always Running. Participant 2 commented, "that was the best experience I've ever had dishwashing! I've always wanted to throw them away" (personal interview 2019). Participant 3 described their personal journey with the dishes as such, "Every time I touch these, I feel the need to clean them again. So, what if I don't clean them and start destroying them?" (personal interview 2019).

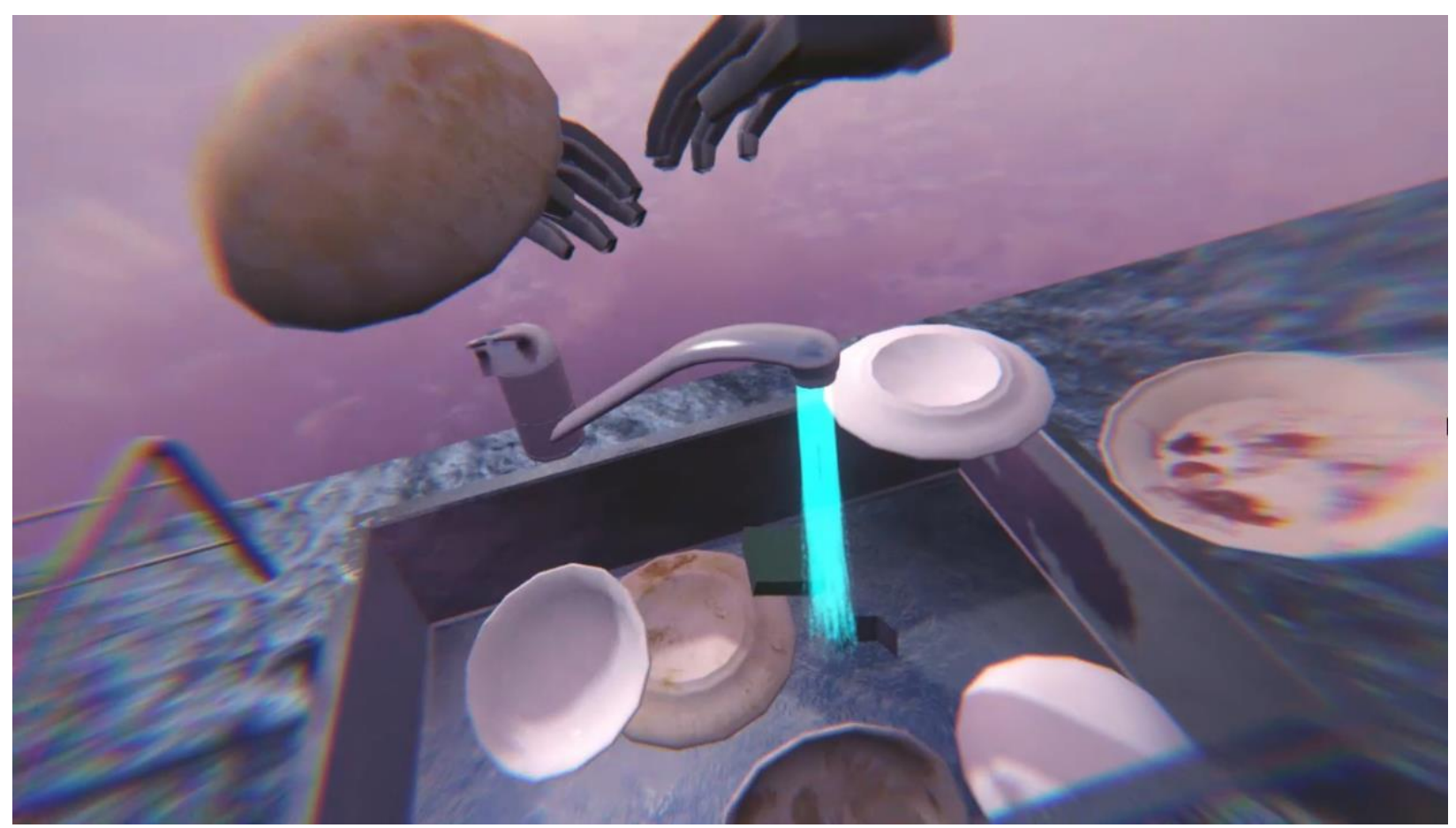

Figure 7. Participant 2 throwing dishes into the air

This subversion of the dishwashing interaction was born for some participants out of what they saw as the futility of accomplishing the dishwashing task. "At first I was trying to do traditional dishwashing," Participant 2 noted (personal interview 2019). This participant also stated, "I don't want to do dishes I want to throw them!" (personal interview 2019) (Figure 7). Participant 3 noted what they called a backwards cycle of clean-not clean that gave birth to a sense of futility. Over the course of Participant 3's time in The Water Is Always Running, they 
realized that, "I have control over this. I'm doing this to myself" (personal interview 2019). Earlier in the interview, when discussing their personal revelations over the course of the experience Participant 3 stated that, “Once I got into this method of recognizing that there wasn't an ending point and everything was this perpetualness (sic), I needed to figure out how to manipulate that" (personal interview 2019). They ultimately came to the conclusion that they could "work through that frustration by chucking the dishes" (personal interview 2019).

\section{Music}

Participants found that the music of The Water Is Always Running was the component of the experience that they felt most tied the interactions to the environment as well as the main draw of the interactions themselves. In discussing their interactions' effect on the overall experience, Participant 5 said, "I think what is cool was the music. It wasn't like I was just interacting with the dishes.” (personal interview 2019). They continued, "It's almost like a puzzle. I was ready to stop. I was just washing the dishes but then I was like wait, no. Every time I pick up a dish it seems like there's a new piece of music that starts playing so now, I'm just going to do that" (personal interview 2019). Participant 1, when discussing the relationship between their actions and the sound said, "My actions were driven by my curiosity surrounding the sound and wanting to know what the next one would be" (personal interview 2019).

Some participants began to actively try and compose with the plates by way of this exploration. Participant 2, in describing their motivations to explore the experience stated, "I could tell different sounds from one [dish] to the other. And then if I was holding three or four you could start making a composition somehow with them" (personal interview 2019). For this participant, their process of composition was also a process of discovery. They continued, “As 
soon as you'd let something go something would come off... grab something and something would come in... So I was playing with that for a while because I was trying to tell what my hands were doing... As soon as I would touch something would change and change and change..." (personal interview 2019). One worthwhile observation from this retelling of the participant's experience is their perception that something would "come off" when they would let go of a plate and something would "come on" when they grabbed a plate. From a technical perspective, the musical loops do not turn on and off based on whether a plate is being held. However, if a plate is clean when picked up the loop will change from its clean state to its dirty state. Also, when a user picks a plate up from the counter, the playback speed increases, resulting in a faster tempo and a higher pitch. This increase in pitch and tempo when a dish is picked up may be significant enough of a differentiator from any other playing dishes to feel as if this dish is actually "coming on."

Feelings of discovery and exploration surrounding the music of The Water Is Always Running was common for many participants. Participant 3 noted, "The first plate I threw off, I could hear sound move away from me... There were moments when the sound would build up... And I don't know if it was to my left, I don't know how exactly I was controlling that" (personal interview 2019). They continued, "I distinctly remember when [the music] started changing, noticing what each plate was doing. Bringing it under the water was changing... things were happening... but not all of it I felt like I could control" (personal interview 2019). Participant 4 described their process as "washing all the dishes to get all the sound out which was obviously what was intended" (personal interview 2019). Participant 6 said, "outside of the water running, every sound was triggered by me from what I could tell... when I put [the dishes] down [the 
sound] either stopped or the sound altered only after I touched them already" (personal interview 2019).

When asked if the environment responded to their actions in a way that was understandable, Participant 1 stated, "Yeah. It was the sound specifically that made everything make sense... the sound always correlated from what I could tell” (personal interview 2019). The last line, "from what I could tell," and other statements shared above play to a trend that seemed to emerge of participants understanding that their actions held influence but not fully understanding the complete extent to which they controlled the music. For many participants, this lack of total understanding seemed to fuel their participation, however. The desire to discover their role within the musical process compelled them to explore and discover ways of interaction beyond what was immediately apparent to them. Participant 1 , in discussing the relationship between their actions and the sound, expressed not feeling "much control of making certain sounds or trying to make it sound good [but] more a curiosity about what would happen" (personal interview 2019). When discussing the same topic Participant 3 expressed a need to “touch everything, combine everything, and try everything" and Participant 2 stated "I think I was trying to compose something because I knew I was trying to add, add, add" (personal interviews 2019).

For Participant 4 in particular, they spoke of developing a more conditioned relationship with the sound as it pertained to their interactions. For most participants, the sound was a sort of vehicle of task emancipation -- that is, these participants began the experience by dishwashing but it was through their discovery of the musical process that they eventually abandoned the dishwashing for more playful and subversive activity, such as throwing the dishes. For Participant 4, they noted that the sound kept them washing dishes. Instead of focusing their 
attention on the musical loops, this participant focused on the single note chime that would play every time a dish was picked up and correlated it to the dish becoming dirty again, stating in the interview, "I would hear the ding and go oh shit it's still dirty" (personal interview 2019). They continued, "every time one of the plates would change there was a sound and I thought this was very Pavlov" (personal interview 2019). When discussing the overall arc of the experience as it related to the sound Participant 4 shared, "Towards the middle/end of my experience I began noticing or begrudgingly hearing the dings of the dishes changing to being dirty again and was not happy" (personal interview 2019). In discussing the way the sounds during cleaning shifted their expectations for their next action this participant said, "I would reach for another dish and hear that stupid ding and go back and start scrubbing or rinsing it again and it moved me back to a task" (personal interview 2019).

The chime when a dish is picked up in The Water Is Always Running was meant to serve as a substitute for the lack of haptic feedback the user experienced when picking up a dish. It was fascinating and quite unexpected -- but entirely understandable in retrospect -- to have a user associate that sound with the dish being dirty. Participant 4 also happened to be the participant that spent the least amount of time overall in the virtual environment, clocking in at just around 5:45 in total. Despite not receiving any instructions on how to interact in the space, Participant 4 seemed very focused on the dishes as their exclusive goal, stating rather succinctly in the post experience interview, "Once I got them all clean and white I took off the headphones" (personal interview 2019). This sense of duty towards the perceived task at hand informed this participants interactions as well, with them stating at one point that, "I tried to change the temperature of the water because I thought if I made it hotter the plates would clean faster, like in real life" (personal interview 2019). 
For a number of participants, the music felt as if it were the bridge between the environment and their interactions. Participant 5 stated that the music, "felt dreamlike, and very fluid almost. Very much like if I was submerged in water that's the kind of music that would be going down. But it synced really well with the world outside of the kitchen which was essentially just sk." (personal interview 2019). When summarizing they said, "The music felt very open and the space was very open" (personal interview 2019). Participant 6 observed that, "the faucet and the sponge kind of made it seem like [I was] in a kitchen, like more realistic. And then the dishes kind of took it to another realm" (personal interview 2019). In describing the relationship between the sound and the environment, Participant 2 humorously noted that, "I don't know if it's any different than when you put on a Bluetooth speaker when you're doing dishes at home... Put on some music to drown out the monotony of the shittiness (sic) of washing dishes" (personal interview 2019).

Participant 3, when asked to describe the relationship between the sound and environment stated, "As I was going back to the complexity of [the sound], it was still really beautiful and pleasing and that seemed to jive with the serenity of the space" (personal interview 2019). As Participant 3 continued answering the question, they made an interesting note about their reaction to hearing a human voice in one of the musical loops:

“...kind of feeling like it was tonal so there was this moment where there were actual words. I forget the repeated phrase but that was interesting because I was in this space by myself and thinking of this as more ambient but then hearing someone's voice singing took me out of this totally alone in another realm by myself feeling" (personal interview 2019). 
This is an interesting insight into the way participants' perception of the environment can be dramatically affected by the nuances of the music. This participant viewed the virtual world of The Water Is Always Running as one belonging exclusively to them and a single musical element reflecting the existence of another person was a seemingly invasive force. Participant 6 , seemingly unbothered by any humanistic qualities in the music, described the dishes as singing, however.

\section{Emotional Responses}

Participants experienced a variety of emotional responses to the various aspects of The Water Is Always Running. The music elicited a number of responses. The participant that felt they were being emotionally conditioned by the chime that played when grabbing a dish is a prime example. Participant 3 described the sounds as very soothing while Participant 1 thought the way the sounds overlapped felt overwhelming and chaotic. Participant 1 did go on to say that the sounds felt normal over time as they grew comfortable in the environment they found themselves in. This same participant also stated being taken aback but also curious about the sound at the beginning and it would seem that this curiosity drove their exploration in spite of their discomfort. In contrast, Participant 3 thought that "the fact that [the sounds] all built harmoniously was really exciting” (personal interview 2019). Later on, Participant 3 also shared a complex emotional arc as it relates to the sound that centered on the faucet, "There was never silence. Always the running water. Always something happening even when I wasn't doing anything there was sound. At first that was peaceful. Then the running water sound became less of a spa and more of 'this water won't shut off kind of thing"” (personal interview 2019). 


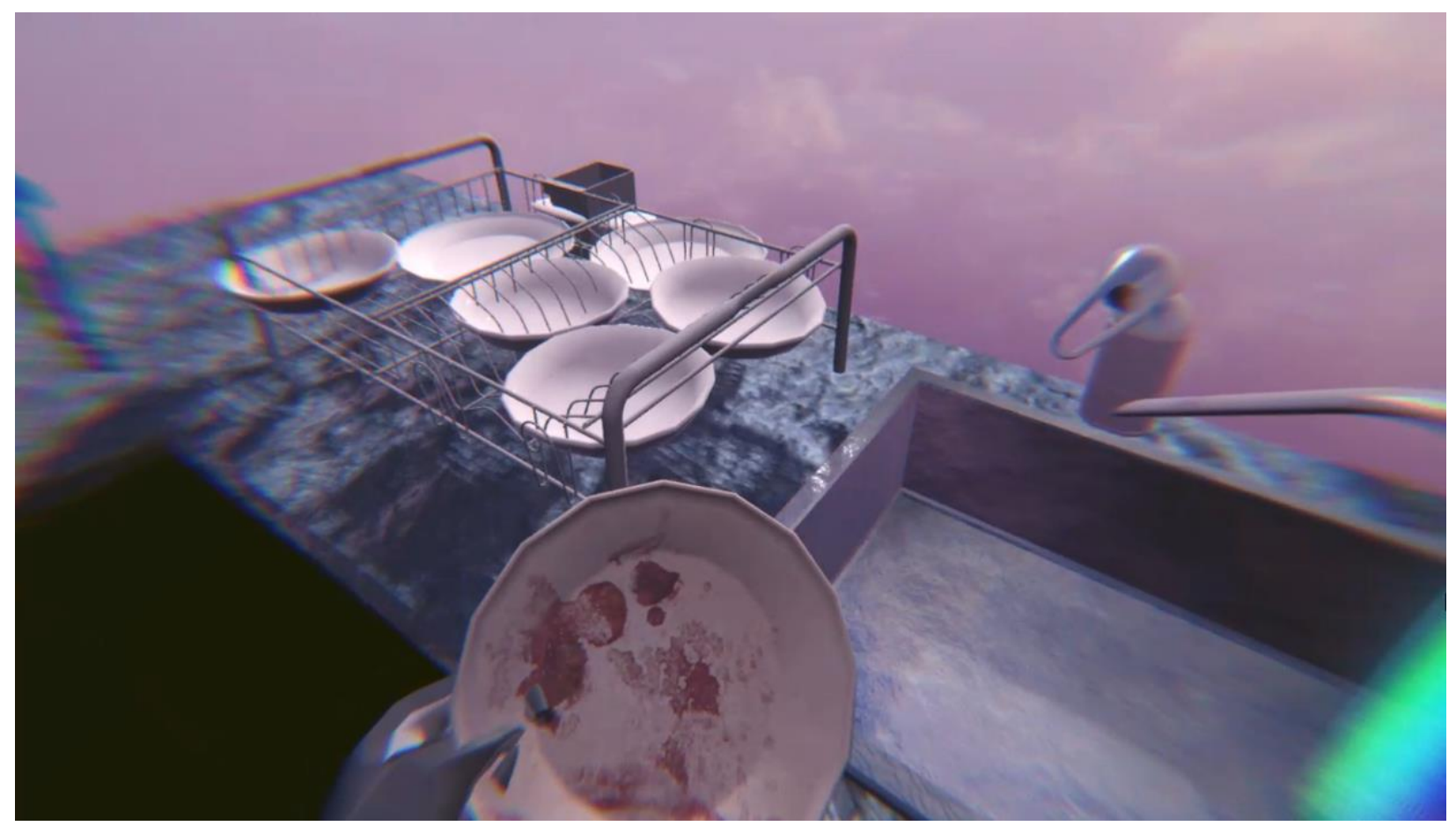

Figure 8. Participant 3 working through the dishes

For Participant 3, the sound of the faucet was a touch point for a deeper emotional response based around real world domestic expectations and the actual futility of completing them in The Water Is Always Running. They stated that, "The thing that was so striking to me was the responsibility of washing the dishes. Like, why was that?" (personal interview 2019). They continued, "I was trying to play with it... I kind of enjoyed playing it but I'm very aware of my forethought being the need to get through the dishes" (personal interview 2019) (Figure 8). They spoke to a direct sense of organization and chaos within the experience and eventually recognizing that the act of dishwashing in The Water Is Always Running was a "cycle [that] was never going to be done" (personal interview 2019). Participant 3 noted the pressure of the faucet as a prime source of their internal conflict stating, "I was so dialed in to this running water and this need to use it because it was constantly running" (personal interview 2019). This 
participant's very first action taken in the experience was attempting to shut the faucet off, stating, "I expected it to turn off. That was my first frustration... my first clue that this wasn't going to go as I wanted it to" (personal interview 2019). This participant eventually concluded they needed to "work through that frustration by chucking the dishes" (personal interview 2019). They called this behavior a "typical angry mom thing" and "something I couldn't do in my home." After this stage, the interaction became focused on exploring the music and became a source of excitement and fun for this participant.

Other emotional responses were centered around the banality of the interaction as it was juxtaposed against the absurdity of the environment and overall premise. One participant noted, “At first I was trying to take it seriously... it wasn't much fun.” For many participants, they felt that the experience did not become fun for them until they stopped trying to take it seriously. In abandoning any attempts to completely clean the dishes or turn the water off and simply explore participants overall seemed to feel a sense of liberation. Overall, participants found the experience as a whole to be a lot with Participant 6 stating, "This is a lot to process" (personal interview 2019).

\section{Overall Experience}

Participants shared a number of interesting responses that can broadly be identified as pertaining to the overall experience as opposed to narrowly fitting into one of the four previously discussed themes. This comes with a certain degree of overlap with the other themes, however. For example, Participant 6, when asked to describe what they had just experienced after exploring The Water Is Always Running, stated, "I was washing dishes spiritually" (personal interview 2019). When expanding upon that sentiment they pointed to the combination of the 
sound and the environment as the cause of their feeling this way. Participant 3 , who was fixated on what they called the "never-ending cycle" of trying to clean the dishes, poignantly shared that The Water Is Always Running, "made me think of the question you asked me 'How often do I do dishes?' How many times a day? Week? Month? Year? That kind of expansion of time if that were condensed down to these ten minutes, I'd be doing that task all the time... thinking about that as my overarching experience" (personal interview 2019). For them, the inability to easily clean all the dishes became this metaphor for the constancy of needing to wash dishes in real life and what it would look like for them to wash a lifetime's worth of dishes in a single setting. This participant eventually worked beyond that sentiment stating that "something I recognized turned into this magical experience" (personal interview 2019).

Participants also shared interesting responses to their overall awareness of the different facets of the experience as well as the shifting nature of the interactions. Participant 2, when asked about whether the sounds they heard during cleaning shifted their expectations for future actions didn't think they were conscious of that, stating, "I felt it was more of an intuitive experience" (personal interview 2019). Participant 3 was consciously thinking about their actions within the experience, stating, " $[I]$ was getting a sense of its perpetual nature and feeling of getting stuck in that cycle was something I was doing to myself" (personal interview 2019). Participant 6 shared, "Now that we're talking about this, the fact that I have a certain way that I do things and that translates into an experience where maybe somebody who doesn't do something I would [would have a different experience] -- is a nice revelation to have" (personal interview 2019). 


\section{CHAPTER V: DISCUSSION}

In creating The Water Is Always Running, I sought to explore the following questions: How can a Fluxus approach to participatory music composition leverage environmental metaphors and affordances? Can a defamiliarized approach to musical interaction heighten the user's awareness of their interactions? How can a meaningful and participatory musical interaction balance between functional and creative user experiences?

This work builds on contemporary research in design principles for visual music and virtual reality musical instruments (Wang 2016) (Serafin et al. 2016). While the research into visual music and VRMIs is foundational to what is being discussed here, it is important to note that the focus of that research is on musical interaction as a means of music making, whereas The Water Is Always Running is musical interaction as a means of music participating and experiencing. But because the shared commonality is a focus on musical interaction in a visual environment, the foundation is fundamentally the same.

Where The Water Is Always Running builds upon the previously discussed research, is in its consideration of the role that the virtual environment has to play in designing a music-led virtual reality. This research probed at the importance of environment by defamiliarizing a common space, a kitchen, and inviting users to subvert their assumed role in that space, as the dishwasher. The results of this study suggested that the environment can play a valuable role in reinforcing the design of music-led virtual realities. In discussing design principles for VRMIs, the authors suggest that designers make use of existing skills (Serafin et al. 2016). In presenting the user with a virtual kitchen and limiting their agency only to the objects commonly used for dishwashing, users were able to quickly discover and confidently understand the basic musical interaction of The Water Is Always Running which then led to playfully exploring how to subvert 
the interaction. This subversion was also supported by the absurdist nature of the environment. The user understands that they are in a kitchen, but they are also aware that they are not in a normal kitchen and that therefore not limited by normative means of conduct within a kitchen.

The emphasis placed on the environment is reflective of the differences between a musicled virtual reality and a virtual reality musical instrument. An instrument is an object to be used and interacted with and is not always dependent upon the space it occupies. Perhaps one of the plates in The Water Is Always Running could be considered an instrument despite its very focused and limited interaction. However, that would ignore the important influence its environment has on the user's understanding of its function. This was even pointed out by one study participant when asked to describe the relationship between the sound and the environment. "That the environment was a kitchen in a sink was a crucial part; if I was sitting on a road with the plates making sounds, I would be doing different things with them," they stated. The affordances of the object are tied to its relationship to the environment. In a music-led virtual reality the depth of both the interaction and the overall experience is vested in how the user participates within the space and not solely within the capabilities of the given object. This chapter presents my key findings from the study and discusses shortcomings and limitations of the system.

\section{Key Findings}

Five key findings have been drawn from the data collected in the user study of The Water Is Always Running. They are presented here as contributions in addendum to the principles presented by Wang and Serafin et. al for any composer looking to design music-led virtual realities. The data collected from user surveys covered topics primarily pertaining to the 
environment, interactions, sounds, their feelings towards, and the overall experience of The Water Is Always Running. That data has been analyzed and these five design principles are suggested in response:

- Converge the functional and the creative

- Subvert expectations

- Design to flip the switch

- Create an acoustic ecology

- User agency is the score/Environment is the instrumentation

These five design principles can be employed to help guide the composer of music-led virtual realities towards the creation of experiences that heighten the user's awareness of process through a defamiliarized approach to musical interaction, balance the functional and creative in meaningful and participatory musical interactions, and leverage environmental metaphors and affordances.

\section{Converge the Functional and the Creative}

Design towards a point of convergence between the functional and conceptual aspects of music-led virtual realities. The strength in the design of The Water Is Always Running is in the synergy present between the design of its interactions and its concept and aesthetic. By settling on an umbrella theme of overconsumption, I was able to use that as bridge when tying disparate components of the overall experience together. Designing towards a point of convergence also kept everything focused. One of Wang's principles for visual music is to simplify and focus on core elements (Wang 2016) and converging every design choice around a core concept helps to 
avoid excess. If an object, interaction, visual element, or audio element does not point back to the central concept then it can be let go of.

Anchoring the functional and creative aspects around a central concept is helpful in creating the bridge between the visual and aural content. One of the earliest hurdles I experienced in designing The Water Is Always Running was to convince any pre-study tester that the audio wasn't just a soundtrack to the dishwashing. This was in part because the interaction lacked salient mapping, as was discussed in Chapter III, but also because at that stage in development, my aesthetic was just functional. The dishwashing metaphor was in place and the music at that point was sampling 80s pop songs but there was little overall connection between these two critical components of the experience. Once overconsumption was settled on as a unifying concept, it became easier to point these two components towards each other. New critical aspects of the experience, such as mapping the playback speed of the music to the $y$-axis to lean into a vaporwave aesthetic or returning the dishes to the counter when thrown off the platform, emerged as a result of designing towards a core concept.

Having a core concept to anchor everything on encourages and bolsters the success rate of potentially risky design choices. Choosing to have dishes become dirty any time a user picks on up, even after it had been cleaned, could have backfired and ruined the experience for participants. However, making it difficult to successfully clean all the dishes lengthened the time participants spent in the virtual environment, added a new level of depth to the interaction, and helped tie the conceptual elements of the experience into the interaction. The more time participants spent in the virtual environment, the more likely they were to explore the experience beyond dishwashing and the more likely they were to begin to focus on the music they were hearing. 


\section{Subvert Expectations}

When employing real life environments as metaphors for musical interactions the greatest opportunities for impact lie in the moments where user's expectations can be subverted. In the case of The Water Is Always Running, this subversion of expectations came through strongest in the moments where the interaction could be defamiliarized. Each of the participant's entered into The Water Is Always Running having had the experience of washing dishes before and therefore possessing an understanding of what affordances are available to them within the kitchen. Participants were most impacted in the moments where they were forced to second guess their assumptions about the space.

One significant point of impact for a number of participants was the fact that they could not turn the faucet off. Participants could interact with the faucet valve, but it would simply spin in place if they did and the water would be left running. In Principles of Visual Design for Computer Music, Wang invites composers to be unafraid of introducing arbitrary constraints into their designs (Wang 2016). It would've been easy to allow participants to turn the faucet off -- in fact, for many participants, being able to shut the water off would've created an overall more pleasant experience. However, by introducing this arbitrary constraint into the design of the dishwashing interaction, it became a flash point for how participants viewed the overall experience. The participants expected to have control over how and when to use the water and, by subverting that expectation, participants felt a certain obligation -- whether informed by social pressures, views on water conservancy, or personal views -- to wash the dishes and, as a result, stay focused on the interaction.

Leaving participants in a kitchen without walls also successfully subverted their expectations and invited participants to grow playful with their interactions. The goal of 
defamiliarization is to heighten a person's awareness of process and/or environment. In removing the walls from the virtual kitchen and placing it within the sky, participants consistently commented that the kitchen and the sky surrounding it felt like two separate environments to them. This defamiliarization of the environment achieved created a sense of presence for the user, something that Serafin et. al advocates for as a principle of design for VRMIs. The participants expected there to be walls surrounding the kitchen and when that expectation was subverted it heightened their overall awareness of their environment. More importantly, it reinforced the overall strangeness of the interaction before participants even picked up a dish which was important information to relay to the participants so that they understood that they also had permission to act strangely as well.

The principle subversion of the interaction was the auditory feedback given to the user when interacting with the dish. The dishwashing interaction was simplified down to its most fundamental gestures: grabbing objects and bringing objects together. This total simplification of the physical interaction meant that participants could easily perform the interaction while their attention could be focused in other places. They could continue to wash dishes if they wanted to while directing their attention to the music being made by their interactions. This subversion of participants' expectations on what a dirty dish or clean dish is supposed to sound like was one of the primary ways in which participants engaged with the experience beyond the act of washing dishes.

When focusing on subverting user expectations as a design goal, composers of music-led virtual realities can lean into their user's understanding of how to participate in an environment and then introduce new modes of thinking and participating in the virtual space by challenging the user's expectations. Designing to subvert expectations is how composers can move user's 
back and forth between normal and magical interactions (Serafin et. al) in a fashion that is discoverable to the user via participation over instruction.

\section{Design to Flip the Switch}

When relying on an environmental metaphor and physical world activities to teach the user interactions, design a way for the user to go beyond the metaphor. One of the most telling data points from this research was that the participant most fixated on the act of dishwashing was the participant that spent the least amount of time experiencing The Water Is Always Running. The dishwashing simulation is not exclusively responsible for that participant's inability to see past the metaphor as the other participant that also fixated intensely on the dishwashing metaphor was the one that spent the most time participating in the experience.

So, the question then, is why did this other participant exclusively see the dishwashing interaction as the totality of the experience? This participant was intently focused while within the experience, noting during their interview that they were unable to even tell how much time they spent in The Water Is Always Running. For this participant, the chime made when a dish was picked up kept drawing their attention back to the dishwashing. The sound designed to be a substitute for haptic feedback became a point of reorientation for the participant, directing them back into the act of dishwashing instead of presenting an opportunity for the participant to see the experience beyond that.

Taking into consideration the fact that dishwashing, while being common place, is often associated as a domestic task to be completed by a female, this issue of time spent within the virtual environment and, beyond that, how much time was spent exclusively on dishwashing could possibly be explained by participants' gender. The data doesn't clearly fall along 
stereotypes in this case. The participant that spent the least amount of time in the virtual environment, Participant 4, was male. However, this participant was also the one that felt conditioned by the sound of the dishes to wash and focused their whole experience exclusively upon that act. This is not clearly contrasted by Participant 3, who is female. Participant 3 spent the most amount of time in the experience, however, this participant successfully moved beyond focusing exclusively on the act of dishwashing.

For the participant that spent the most time in The Water Is Always Running there was a moment during their experience where they decided to stop taking everything so seriously. For them, there was a moment where they flipped the switch on their own experience. They stopped seeing the experience as being just a dishwashing simulation and began to see it for everything it was beyond that. Perhaps Participant 3, in flipping the switch, chose to redefine the domestic lexicon present in The Water Is Always Running for themselves reminiscent in the same fashion as Semiotics of the Kitchen. This participant noted the futility of being able to successfully wash all the dishes and feeling trapped in a never-ending cycle -- not unlike how the other participant felt. Whereas, Participant 4 felt the sound drew them back into dishwashing, Participant 3 felt that it was the part to focus their attention on after recognizing the futility of trying to wash all the dishes.

It is important for composers of music-led virtual realities to design towards flipping that switch. Part of this is achieved by subverting user's expectations. This was successful in the case of the Participant 3. In the case of Participant 4 a certain factor of the interaction ultimately reinforced their expectations. Designing to flip the switch is anticipating how your user will cross the threshold between understanding the intended interaction and experiencing the intended experience. This is, of course, not to say that Participant 4 was wrong in their experience because 
they were not. Ultimately this is illustrative of the point discussed in Chapter III surrounding the conflict that can arise between authorial intent and user experience. The intent was for the user to see the dishwashing metaphor as a means of exploring the musical interactions available between themselves, the dishes, and the environment. When relying on a metaphor such as dishwashing to teach interactions to the user, it is important to design towards the moment the user moves past that metaphor.

\section{Create an Acoustic Ecology}

When creating a music-led virtual reality, it is important to consider the acoustic ecology of the virtual environment. Much in the same way that users will approach objects in a virtual environment the same way that they would in the physical environment, they will also approach sound in a virtual environment the same way they would a physical environment. Acoustic ecology was developed as a way to understand the relationship between a person and their sonic environment. Breaking down a music-led virtual reality using acoustic ecology terminology is effective in understanding how users might perceive the sonic environment created by the experience. It is also useful for analyzing an already existing music-led virtual reality.

The hierarchy of the virtual soundscape of The Water Is Always Running can be understood when broken down using acoustic ecology terminology. Central to every soundscape is the keynote, or the sound against which all other sounds are measured. In the case of The Water Is Always Running, the sound of the faucet was the keynote for the whole experience. It is the first sound the user hears in the experience and it is the only sound they cannot control. It's position as the keynote is also likely informed by the fact that it is one of the few sounds in the soundscape whose placement is logical to the user. If there is a faucet running, those who are 
able expect to hear it. Because the sound of the running water is an expected one, it provides the necessary contrast to the more fantastical sounds of the plates.

In considering the function of the dishes within the overall soundscape, an individual dish is a sound signal while the dishes collectively are a sound event. Sound signals are defined simply as any sound meant to be listened to whereas a sound event is a sound or sequence that gains meaning through its social and environmental context. The sound of an individual dish is the first significant sound a user hears beyond the sound of the running water. Participants consistently expressed surprise and curiosity over the sounds of the dishes - particularly when hearing one for the first time. Participants understood the inherent significance of their sound. It was when participants began exploring multiple dishes simultaneously that the soundscape began to have context for many of them and create a sound event. Participant 2, for example, was focused on exploring the relationships between the dishes in order to create a composition. This dramatically affected the overall environment as well as shifted their perception of the entire experience by providing context to each dish.

Beyond simply categorizing the role of the dishes in the overall soundscape, the sound of the dishes can be categorized based on how the participants felt about the sound. Because the music is partially created using sampled pop songs from the 1980 s, there are added layers to how the participants might have interpreted the sounds. In acoustic ecology there are four terms that can help to provide context to the music: disappearing sound, sacred noise, sound romance, and moozak. The fact that the music sampled is $30+$ year old pop songs would qualify the music as disappearing sounds or sounds that were once heard commonly but are no longer. The participants may experience a sound romance, or a nostalgic remembering, with the music. Participant 3, for example, spent time fiddling with the placement of a dish in their hands 
because they believed they heard Sting's voice and wanted to find the right playback speed to confirm their suspicions. On a more nuanced level, this familiarity with the music could be because pop music -- dating well before the 1980s -- is sacred noise, or sound exempt from social prescription. In the case of pop songs, it's often in the form of moozak meant to soundtrack shopping experiences -- a point that ties back into the conceptual focus of The Water Is Always Running on overconsumption. When sampling popular pieces of music, it's important to remember that users will bring their own context into the new context provided for them.

Another important concept to acoustic ecology which can be applied to music-led virtual realities like The Water Is Always Running is the idea of a hi-fi soundscape vs a lo-fi soundscape. These two concepts are roughly analogous to the idea of arrangement in traditional musical forms. A hi-fi soundscape is one with little sonic overlap and greater perspective and spatial awareness. In acoustic ecology, hi-fi soundscapes are most found in nature and are the ideal. A lo-fi soundscape is the opposite; a densely packed soundscape with little perspective and spatial awareness. Industrial environments are frequently cited as examples. Certain participants noted the fluctuating density of the soundscape in The Water Is Always Running and their remarks reflected the general qualities of each soundscape. When one or two dishes were playing, participants were able to better understand information such as spatialization and from which dish emanated which sound. As more dishes were activated, the overall soundscape became more sonically rich and interesting but individual components were more difficult to distinguish.

It is important to consider how the soundscape would be analyzed by potential users during the design phase, even though an analysis like this is best produced when having ears other than the creator's listening to the soundscape. In planning for the overall soundscape, the composer of a music-led virtual reality can anticipate how users will perceive individual 
elements within the soundscape as well as predict possible arrangements of the soundscape, their impact on the user, and how the user is able to transform the soundscape between hi-fi and lo-fi.

\section{User Agency is the Score / Environment is the Instrumentation}

The difficulty with creating music-led virtual realities is in translating the ideas of a musical composition into spatial terms that give priority to user agency. When creating a musicled virtual reality, the composer's control of the overall musical output ends where the user's agency in the environment begins. In the case of The Water Is Always Running, I was able to control what sounds interactable objects made and how those sounds were mapped to those objects. While that was the limitations by which I could directly influence the final musical output of the overall experience, I was still able to exert influence by way of user agency and environmental affordances. For example, by removing the ability of the user to be able to turn off the water, I was able to guarantee a sonic foundation that also encouraged the user to introduce other sounds by way of leveraging the social tension from needing to do something with the running water. This is the same thinking behind why a dish returns to the counter when thrown off the platform. The purpose of this experience is for the user to participate in the musical process and, therefore, it would be counterintuitive to allow the user to permanently remove musical elements with no means to retrieve them. In forcing the dishes back into the interactable space, the user still has the freedom to interact with the dish however they'd like without taking the experience outside of the intended bounds.

It is helpful to think of this relationship as such: user agency is the score and the environment is the instrumentation. In traditional forms of music, the score consists of patterns of notes sequenced together over a duration of time in a linear fashion. In a fluxus event score, it 
is the list of instructions to be carried out -- sometimes linearly, other times not. In a music-led virtual reality, the score is the entirety of possible actions the user can take. When those possible actions are presented in a linear fashion, it is still closer to an event score in that it is guided participation as an act of performance. The score is not a set of instructions but instead a list of anticipated possible outcomes, or by looking at it from a different angle, a list of arbitrary constraints -- what the user can't do in a given space.

If user agency is the score, then the environment and all interactable objects within it are the instrumentation. For example, a marimba, snare drum, and berimbau are all played by striking the instrument with something, but the object used for striking and the sound produced is different for all three. This same idea translates to the function of the environment and objects in a music-led virtual reality. A user can perform a simple grabbing action but the object that they are grabbing and the environment in which they grab it will influence their participation. This can be used to the composer's advantage to create new textures and timbres for the user to experience based on the way they perceive their participation within the space.

Earlier, it was discussed that an individual reflects upon the environment within which they find themselves and through that reflection, using their own metaphorical and perceptual models, they begin to understand their own agency within a space. A composer of music-led virtual realities, in leveraging those metaphorical and perceptual models, can influence the user's perception of their own agency within the virtual environment. In doing so, the composer can make informed presumptions about how the user will participate within the space and design th environments and interactions accordingly. In the case of The Water Is Always Running, by leaning into the user's perceptual model surrounding the kitchen, I could anticipate how they would approach objects within the environment and assign musical functions accordingly. In this 
way, I was laying the groundwork for potential scores for users through leaning into these metaphors and understanding that the environment would influence their perception of the content much in the same way that a choice of instrument influences the perception of a melody.

\section{Limitations and Future Works}

It is important to note that there are limitations within the study conducted and, as a result, the data collected through it. Participants only had a single session within the music-led virtual reality during their time sitting for an interview. While this freeform session was valuable for gathering data on how participants perceived the environment and its affordances, having a second, guided session could have corroborated the first session's results and provided an opportunity to further gauge participant's understanding of the interactions available to them. Likewise, having a larger sample group could have yielded a broader data set. However, the consistency of participants' responses surrounding the environment, sound, interaction, and overall experience provides sufficient evidence to warrant the key findings while also accommodating for any noteworthy discrepancies as well.

There were shortcomings in the design that will be addressed in future iterations. Participants had a difficult time understanding how to get a dish to stop playing as a result of the lack of slotting functionality within the drying rack. Dishes can only be set on top of the drying rack. Surrounding the drying rack is a collider that turns off whatever dish enters it. However, participants were expecting the virtual drying rack to have the same affordances as a real one and, as a result, when the dish would fall over in the drying rack, they would consistently go to pick it up and try to slot it in, thus causing the dish to return to its dirty state and the music to 
begin again. Ultimately, this meant the participants were unaware of the mapping afforded to the drying rack.

In subsequent iterations of the software, I would also like to find a way to greater incentivize throwing dishes off of the platform as a way of helping to guide users beyond the dishwashing and exploring other modes of participation. I believe this can be achieved by including interactable objects located off of the platform where participants stand that can be activated by coming in contact with a dish. The data reflects that most participants were interested in the space beyond the platform and by placing objects within that space could act as incentives. This would also help to teach the user that the dish would return to the counter upon being thrown. The safety of knowing the dish would return to the counter would help to counteract any social taboos a user might feel about throwing dishes and encourage more exploratory interactions. The appearance of these objects within the space beyond the platform would need to be a timed event to ensure that they occur after the user has been given enough time to fully grasp the dishwashing interaction and the relationship between the sound and objects in the environment. 


\section{CHAPTER VI: CONCLUSION}

This thesis examines how a Fluxus approach to participatory music can leverage environmental metaphors and affordances, heighten user awareness of their interactions through a defamiliarized approach to musical interaction, and balance functional and creative user experiences in a meaningful and participatory musical interaction. This research has been conducted using the virtual reality experience The Water Is Always Running. This work presents an unusual music-making environment, a 3D kitchen with dishwashing simulation, to explore how a defamiliarized approach to musical interaction and participation can heighten the awareness of process for the user.

The goal of this process was to create a music-led virtual reality that explored the idea of an embodied musical composition that was meant to be experienced by way of participation, test it with users, and establish a blueprint for future works. Each participant took part in a half hour session that consisted of a section of establishing questions, followed by an open-ended period of exploration in The Water Is Always Running, and finishing with an interview focused on the participants feelings and perception of the experience.

The results of the study reflected the usefulness of leveraging environmental metaphors and affordances to heighten awareness of musical interaction, such as: the successful use of interactions common to everyday life as a vehicle to create discoverable musical interactions, the ability to create and anticipate moments of significance to the user by subverting expectations of the environment and processes present within it, and ensuring the musical experience was not subservient to the visual experience by designing both around a unified concept. Participants were asked questions related to their feelings about the experience, their expectations as they explored the experience, their personal analysis of the experience, and a number of questions 
probing the metaphors and affordances of the experience. Participants shared a wide range of meaningful responses pertaining to the environment, interactions, sound, their emotions, and the overall experience of The Water Is Always Running.

This research was limited in its sample size and the number of opportunities users had to participate in the experience outside of each user's freeform session of exploration. Future inquiries into the design of music-led virtual realities would benefit from increased user exposure to tested experiences as well as guided sessions to probe deeper into specific areas of inquiry within the research. In future iterations of The Water Is Always Running the virtual drying rack will be updated to add a slotting mechanism that is in line with user expectations for the device to have the same affordances as its physical counterpart. There will also be the addition of interactable objects beyond the platform that can only be activated by throwing dishes as a means to help guide the player beyond the dishwashing activity to encourage extended exploration.

This research builds on a set of foundational principles for visual design for computer music and principles for design of virtual reality musical instruments by offering for consideration the inclusion of five additional design principles for creating music-led virtual realities: converge the functional and creative, subvert expectations, design to flip the switch, create an acoustic ecology, and user agency is the score/environment is the instrumentation. 


\section{REFERENCES}

Ableton. (2020). Ableton. Retrieved March 9, 2020, from https://www.ableton.com/

Audio-first VR: Imagining the Future of Virtual Environments for Musical Expression. (2018).

Retrieved March 9, 2020, from https://audio1stvr.github.io/

Audiokinetic Inc. (2020). Wwise. Retrieved March 9, 2020, from

https://www.audiokinetic.com/products/wwise/

Auslander, P. (n.d.). Fluxus art-amusement: The future of music? Art Papers, (23), 30-35.

Baudrillard, J. (1994). Simulacra and simulation. University of Michigan press.

Buckley, Z., \& Carlson, K. (2019). Towards a Framework for Composition Design for Music-

Led Virtual Reality Experiences. 2019 IEEE Conference on Virtual Reality and 3D User

Interfaces (VR). doi: 10.1109/vr.2019.8797768

Buckley, Z. (2020). The Water Is Always Running (VR). Retrieved March 9, 2020, from https://zwbuckley.itch.io/the-water-is-always-running

Cheng, H., \& Liu, S. (2019). Haptic Force Guided Sound Synthesis in Multisensory Virtual Reality (VR) Simulation for Rigid-Fluid Interaction. 2019 IEEE Conference on Virtual Reality and 3D User Interfaces (VR). doi: 10.1109/vr.2019.8797906

Chon, S. H., \& Kim, S. (2019). The Matter of Attention and Motivation - Understanding Unexpected Results from Auditory Localization Training Using Augmented Reality. 2019 IEEE Conference on Virtual Reality and 3D User Interfaces (VR). doi:

$10.1109 /$ vr.2019.8797683

Chuck Person's Eccojams vol.1 : Chuck Person. (2010, August 8). Retrieved March 9, 2020, from https://archive.org/details/svhscr_protonmail_03A3 
Cook, P. (2017). 2001: Principles for Designing Computer Music Controllers. A NIME Reader Current Research in Systematic Musicology, 1-13. doi: 10.1007/978-3-319-47214-0_1 deliriously...daniel. (2019). As the Spirit Moves: An Interview with 회사AUTO. Private Suite Magazine, (6), 48-53.

Electronic Arts Intermix. (2006). Semiotics of the Kitchen. Retrieved March 9, 2020, from https://web.archive.org/web/20061006090705/http://www.eai.org/eai/tape.jsp?itemID=15 45

Far Side Virtual: James Ferraro. (2020, February 17). Retrieved March 9, 2020, from https://archive.org/details/farsidevirtual/

FAVR 2019: Future of Audio in Virtual Reality. (2019). Retrieved March 9, 2020, from https://favr2019.github.io/

Floral Shoppe, by Macintosh Plus. (2011, December 9). Retrieved March 9, 2020, from https://vektroid.bandcamp.com/album/floral-shoppe

Friedman, K., Smith, O. F., \& Sawchyn, L. (Eds.). (2002). The Fluxus performance workbook. Performance Research e-Publications.

Glitsos, L. (2017). Vaporwave, or music optimised for abandoned malls. Popular Music, 37(1), 100-118. doi: 10.1017/s0261143017000599

Hamilton, R. (2018, November 4). Retrieved from https://www.youtube.com/watch?v=cgODPY90pAU\&feature=youtu.be Hamilton, R. (2014). Perceptually Coherent Mapping Schemata for Virtual Space and Musical Method. Stanford University.

Hamilton, R. (2013). ECHO::Canyon. Retrieved March 22, 2020, from https://ccrma.stanford.edu/ rob/portfolio/echocanyon/ 
Hunt, A., Wanderley, M. M., \& Paradis, M. (2AD). The importance of parameter mapping in electronic instrument design. Proceedings of the 2002 Conference on New Instruments for Musical Expression.

Illinois State University. (2019). Planning, Research, and Policy Analysis: Census Day: Enrollment Report: Spring 2019. Illinois State University.

Jerald, J. (2016). The Vr book: human-centered design for virtual reality. New York: Association for Computing Machinery.

JetPuffed. (2018). Vaporwave's Complicated Relationship with Consumerism and Capitalism. Private Suite Magazine, (1), 1-4.

Johnston, A., Candy, L., \& Edmonds, E. (2008). Designing and evaluating virtual musical instruments: facilitating conversational user interaction. Design Studies, 29(6), 556-571. doi: 10.1016/j.destud.2008.07.005

Jordà, S. (2004). Instruments and Players: Some Thoughts on Digital Lutherie. Journal of New Music Research, 33(3), 321-341. doi: 10.1080/0929821042000317886

Killeen, P. (2018). Burned Out Myths and Vapour Trails: Vaporwave's Affective Potentials. Open Cultural Studies, 2(1), 626-638. doi: 10.1515/culture-2018-0057

Lakoff, G., \& Johnson, M. (1980). Metaphors We Live By. Humans as Symbolic Creatures, 124-134.

Liss, A. (2009). Feminist art and the maternal. Minneapolis: University of Minnesota Press. Machover, T. (2002). Instruments, Interactivity, and Inevitability. Proceedings of the NIME International Conference. 
Men, L., \& Bryan-Kinns, N. (2018). LeMo: Supporting Collaborative Music Making in Virtual Reality. 2018 IEEE 4th VR Workshop on Sonic Interactions for Virtual Environments (SIVE). doi: 10.1109/sive.2018.8577094

Merleau-Ponty, M., \& Landes, D. A. (2012). Phenomenology of perception. Abingdon, Oxon: Routledge.

Mäki-Patola, T., Laitinen, J., Kanerva, A., \& Takala, T. (2005). Experiments with Virtual Reality. Proceedings of the 2005 International Conference on New Interfaces for Musical Expression.

Neely, A. (2016, October 24). The music theory of V A P O R W A V E. Retrieved March 9, 2020, from https://www.youtube.com/watch?v=QdVEez20X_s

Norman, D. A. (1988). The Design of Everyday Things. London: Basic.

Paine, G. (2015). Interaction as Material: The techno-somatic dimension. Organised Sound, 20(1), 82-89. doi: 10.1017/s1355771814000466

Pulkki, V., Politis, A., Pihlajamäki, T., \& Laitinen, M.-V. (2017). Spatial Sound Scene Synthesis and Manipulation for Virtual Reality and Audio Effects. Parametric Time-Frequency Domain Spatial Audio, 347-361. doi: 10.1002/9781119252634.ch14

Schafer, R. M. (1977). The Tuning of the World. New York City, NY: Knopf.

Schiettecatte, B. (2004). Interaction design for electronic musical interfaces. Extended Abstracts of the 2004 Conference on Human Factors and Computing Systems - CHI 04. doi: $10.1145 / 985921.986125$

Serafin, S., Erkut, C., Kojs, J., Nordahl, R., \& Nilsson, N. C. (2016). Virtual reality musical instruments. Proceedings of the Audio Mostly 2016 on - AM 16. doi: $10.1145 / 2986416.2986431$ 
Shklovsky, V. (2015). Art, as Device. Poetics Today, 36(3), 151-174. doi: 10.1215/033353723160709

Sonic Interactions in Virtual Environments. (2020). Retrieved March 9, 2020, from https://sive.create.aau.dk/

Sreng, J., \& Lécuyer, A. (2013). Simulating Contacts between Objects in Virtual Reality with Auditory, Visual, and Haptic Feedback. Sonic Interaction Design. doi: 10.7551/mitpress/8555.003.0021

Summers, C., Lympouridis, V., \& Erkut, C. (2015). Sonic interaction design for virtual and augmented reality environments. 2015 IEEE 2nd VR Workshop on Sonic Interactions for Virtual Environments (SIVE). doi: 10.1109/sive.2015.7361290

Summers, C., \& Jesse, M. (2017). Creating immersive and aesthetic auditory spaces in virtual reality. 2017 IEEE 3rd VR Workshop on Sonic Interactions for Virtual Environments (SIVE). doi: 10.1109/sive.2017.7938144

The University of Chicago. (2012, March 28). Alison Knowles: Fluxus Event Scores. Retrieved March 9, 2020, from https://www.youtube.com/watch?v=064qvwX_-kA

Ultraleap. (2020). Leap Motion Controller. Retrieved March 9, 2020, from https://www.ultraleap.com/

Unity Technologies. (2020). Unity. Retrieved March 9, 2020, from https://unity.com/ Villegas, J. (2015). Locating virtual sound sources at arbitrary distances in real-time binaural reproduction. Virtual Reality, 19(3-4), 201-212. doi: 10.1007/s10055-015-0278-0

Voong, T. M., \& Oehler, M. (2019). Auditory Spatial Perception Using Bone Conduction Headphones along with Fitted Head Related Transfer Functions. 2019 IEEE Conference on Virtual Reality and 3D User Interfaces (VR). doi: 10.1109/vr.2019.8798218 
Wang, G. (2016). Some Principles of Visual Design for Computer Music. Leonardo Music Journal, 26(26), 14-19. doi: 10.1162/lmj_a_00960

Wozniewski, M., Settel, Z., \& Cooperstock, J. (2013). Sonic Interaction via Spatial Arrangement in Mixed-Reality Environments. Sonic Interaction Design. doi: 10.7551/mitpress/8555.003.0022

Wrightson, K. (2000). An introduction to acoustic ecology. Soundscape: The Journal of Acoustic Ecology, 1(1), 10-13. 


\section{APPENDIX A: INTERVIEW QUESTIONS ASKED TO STUDY PARTICIPANTS}

Pre-Experience Questions:

1. How many domestic tasks do you complete in a day/week?

2. How often do you do dishes?

3. Have you used virtual reality before? If so, how frequently?

Post-Experience Questions

1. How do you feel about what you just experienced?

2. Can you describe what you just experienced for me?

3. What did you want to do first in the experience?

4. What did you expect to happen when you did your first action?

5. How did the scenario change over time?

6. Did your actions affect the experience and, if so, how?

7. Were the sounds made during your cleaning expected? Did you anticipate any of the sounds you heard?

8. How did the sounds during your cleaning shift your expectations for the next action you wanted to do?

9. What was the most compelling part of the experience for you?

10. How would you describe the relationship between the sound and the environment?

11. How would you describe the relationship between your action and the sounds?

12. Do you feel the environment responded to your actions in a way that was understandable to you? 